\title{
Closing Numbers and Super-Closing Numbers as (Dual)Resolving and (Dual)Coloring alongside (Dual)Dominating in (Neutrosophic)n-SuperHyperGraph
}

\author{
Henry Garrett \\ Independent Researcher \\ DrHenryGarrett@gmail.com \\ Twitter's ID: @DrHenryGarrett | @DDrHenryGarrett.wordpress.com
}

\begin{abstract}
New setting is introduced to study "closing numbers" and "super-closing numbers" as optimal-super-resolving number, optimal-super-coloring number and optimal-super-dominating number. In this way, some approaches are applied to get some sets from (Neutrosophic)n-SuperHyperGraph and after that, some ideas are applied to get different types of super-closing numbers which are called by optimal-super-resolving number, optimal-super-coloring number and optimal-super-dominating number. The notion of dual is another new idea which is covered by these notions and results. In the setting of dual, the set of super-vertices is exchanged with the set of super-edges. Thus these results and definitions hold in the setting of dual. Setting of neutrosophic n-SuperHyperGraph is used to get some examples and solutions for two applications which are proposed. Both setting of SuperHyperGraph and neutrosophic n-SuperHyperGraph are simultaneously studied but the results are about the setting of n-SuperHyperGraphs. Setting of neutrosophic n-SuperHyperGraph get some examples where neutrosophic hypergraphs as special case of neutrosophic n-SuperHyperGraph are used. The clarifications use neutrosophic n-SuperHyperGraph and theoretical study is to use n-SuperHyperGraph but these results are also applicable into neutrosophic n-SuperHyperGraph. Special usage from different attributes of neutrosophic n-SuperHyperGraph are appropriate to have open ways to pursue this study. Different types of procedures including optimal-super-set, and optimal-super-number alongside study on the family of (neutrosophic)n-SuperHyperGraph are proposed in this way, some results are obtained. General classes of (neutrosophic)n-SuperHyperGraph are used to obtains these closing numbers and super-closing numbers and the representatives of the optimal-super-coloring sets, optimal-super-dominating sets and optimal-super-resolving sets. Using colors to assign to the super-vertices of n-SuperHyperGraph and characterizing optimal-super-resolving sets and optimal-super-dominating sets are applied. Some questions and problems are posed concerning ways to do further studies on this topic. Using different ways of study on n-SuperHyperGraph to get new results about closing numbers and super-closing numbers alongside sets in the way that some closing numbers super-closing numbers get understandable perspective. Family of n-SuperHyperGraph are studied to investigate about the notions, super-resolving and super-coloring alongside super-dominating in n-SuperHyperGraph. In this way, sets of representatives of optimal-super-colors, optimal-super-resolving sets and optimal-super-dominating sets have key role.
\end{abstract}


Optimal-super sets and optimal-super numbers have key points to get new results but in some cases, there are usages of sets and numbers instead of optimal-super ones. Simultaneously, three notions are applied into (neutrosophic)n-SuperHyperGraph to get sensible results about their structures. Basic familiarities with n-SuperHyperGraph theory and neutrosophic n-SuperHyperGraph theory are proposed for this article.

Keywords: Coloring Numbers, Resolving Numbers, Dominating Numbers AMS Subject Classification: 05C17, 05C22, $05 \mathrm{E} 45$

\section{Background}

Fuzzy set in Ref. [16], neutrosophic set in Ref. [2], related definitions of other sets in Refs. [2, 13, 15], graphs and new notions on them in Refs. [5-11], neutrosophic graphs in Ref. [3], studies on neutrosophic graphs in Ref. [1], relevant definitions of other graphs based on fuzzy graphs in Ref. [12], related definitions of other graphs based on neutrosophic graphs in Ref. [4], are proposed.

In this section, I use two subsections to illustrate a perspective about the background of this study.

\subsection{Motivation and Contributions}

In this study, there's an idea which could be considered as a motivation.

Question 1.1. Is it possible to use mixed versions of ideas concerning "super-domination", "super-dimension" and "super-coloring" to define some super-notions which are applied to n-SuperHyperGraph?

It's motivation to find notions to use in any classes of n-SuperHyperGraph. Real-world applications about time table and scheduling are another thoughts which lead to be considered as motivation. Connections amid two items have key roles to assign super-colors, super-domination and super-dimension. Thus they're used to define new super-ideas which conclude to the structure of super-coloring, super-dominating and super-resolving. The concept of having general super-edge inspires me to study the behavior of general super-edge in the way that, three types of "super-closing" numbers, e.g., super-coloring numbers, super-dominating numbers and super-resolving numbers are the cases of study in the settings of individuals and in settings of families.

The framework of this study is as follows. In the beginning, I introduced basic definitions to clarify about preliminaries. In section "New Ideas For n-SuperHyperGraph", new notions of super-coloring, super-dominating and super-resolving are applied to super-vertices of SuperHyperGraph as individuals. In section "Optimal Numbers For n-SuperHyperGraph", specific closing numbers have the key role in this way. Classes of n-SuperHyperGraph are studied in the terms of different closing numbers in section "Optimal Numbers For n-SuperHyperGraph" as individuals. In the section "Optimal Sets For n-SuperHyperGraph", usages of general sets and special sets have key role in this study as individuals. In section "Optimal Sets and Numbers For Family of n-SuperHyperGraph", both sets and closing numbers have applied into the family of n-SuperHyperGraph. In section "Applications in Time Table and Scheduling", two applications are posed for n-SuperHyperGraph concerning time table and scheduling when the suspicions are about choosing some subjects. In section "Open Problems", some problems and questions for further studies are proposed. In section "Conclusion and Closing Remarks", gentle discussion about results and applications are featured. In section "Conclusion and Closing Remarks", a brief 
overview concerning advantages and limitations of this study alongside conclusions are formed.

\subsection{Preliminaries For Setting of Neutrosophic n-SuperHyperGraph}

Definition 1.2. (Graph).

$G=(V, E)$ is called a graph if $V$ is a set of objects and $E$ is a subset of $V \times V(E$ is a set of 2-subsets of $V$ ) where $V$ is called vertex set and $E$ is called edge set.

Every two vertices have been corresponded to at most one edge.

Definition 1.3. (Hypergraph).

$H=(V, E)$ is called a hypergraph if $V$ is a set of objects and for every nonnegative integer $t \leq n, E$ is a set of $t$-subsets of $V$ where $V$ is called vertex set and $E$ is called hyperedge set.

Definition 1.4. (Neutrosophic Hypergraph).

$N H G=\left(V, E, \sigma=\left(\sigma_{1}, \sigma_{2}, \sigma_{3}\right), \mu=\left(\mu_{1}, \mu_{2}, \mu_{3}\right)\right)$ is called a neutrosophic hypergraph if it's hypergraph, $\sigma_{i}: V \rightarrow[0,1], \mu_{i}: E \rightarrow[0,1]$, and for every $v_{1} v_{2} \cdots v_{t} \in E$,

$$
\mu\left(v_{1} v_{2} \cdots v_{t}\right) \leq \sigma\left(v_{1}\right) \wedge \sigma\left(v_{2}\right) \wedge \cdots \sigma\left(v_{t}\right) .
$$

$(i): \sigma$ is called neutrosophic vertex set.

(ii) : $\mu$ is called neutrosophic hyperedge set.

(iii) : $|V|$ is called order of NHG and it's denoted by $\mathcal{O}(N H G)$.

$(i v): \Sigma_{v \in V} \sigma(v)$ is called neutrosophic order of NHG and it's denoted by $\mathcal{O}_{n}(N H G)$.

$(v i):|E|$ is called size of NHG and it's denoted by $\mathcal{S}(N H G)$.

(vii) : $\Sigma_{e \in E} \mu(e)$ is called neutrosophic size of NHG and it's denoted by $\mathcal{S}_{n}(N H G)$.

Example 1.5. Assume Figure (7).

$(i)$ : Neutrosophic hyperedge $n_{1} n_{2} n_{3}$ has three neutrosophic vertices.

(ii) : Neutrosophic hyperedge $n_{3} n_{4} n_{5} n_{6}$ has four neutrosophic vertices.

(iii) : Neutrosophic hyperedge $n_{1} n_{7} n_{8} n_{9} n_{5} n_{6}$ has six neutrosophic vertices.
$(i v): \sigma=\left\{\left(n_{1},(0.99,0.98,0.55)\right),\left(n_{2},(0.74,0.64,0.46)\right),\left(n_{3},(0.99,0.98,0.55)\right)\right.$, $\left(n_{4},(0.54,0.24,0.16)\right),\left(n_{5},(0.99,0.98,0.55)\right),\left(n_{6},(0.99,0.98,0.55)\right)$, $\left.\left.\left(n_{7},(0.99,0.98,0.55)\right),\left(n_{8},(0.99,0.98,0.55)\right),\left(n_{9},(0.99,0.98,0.55)\right)\right\}\right)$ is neutrosophic vertex set.

$$
\begin{aligned}
(v): & \left.\mu=\left\{\left(e_{1},(0.01,0.01,0.01)\right),\left(e_{2},(0.01,0.01,0.01)\right),\left(e_{3},(0.01,0.01,0.01)\right)\right\}\right) \text { is } \\
& \text { neutrosophic hyperedge set. } \\
(v i): & \mathcal{O}(N H G)=9 . \\
(v i i): & \mathcal{O}_{n}(N H G)=(8.21,7.74,4.47) . \\
(\text { viii }): & \mathcal{S}(N H G)=3 . \\
(i x): & \mathcal{S}_{n}(N H G)=(0.03,0.03,0.03) .
\end{aligned}
$$




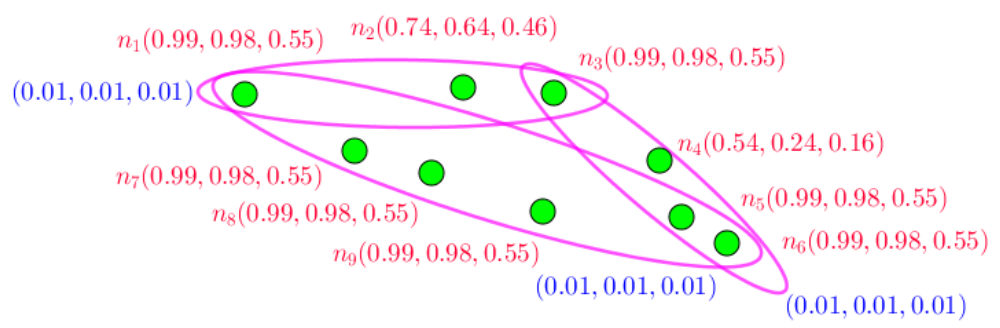

$N H G$

Figure 1. There are three neutrosophic hyperedges and two neutrosophic vertices.

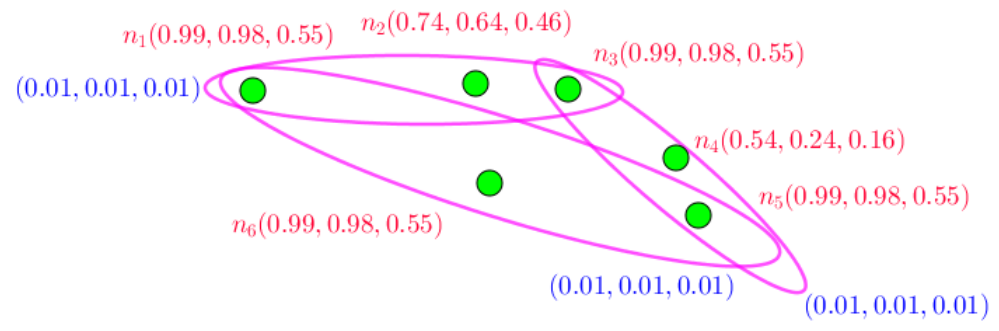

NHG

Figure 2. $N H G=(V, E, \sigma, \mu)$ is neutrosophic edge 3-regular hypergraph

Definition 1.6. (Neutrosophic Edge $t$-Regular Hypergraph).

A neutrosophic hypergraph $N H G=(V, E, \sigma, \mu)$ is called a neutrosophic edge $t$-regular hypergraph if every neutrosophic hyperedge has only $t$ neutrosophic vertices.

Question 1.7. What-if all neutrosophic hypergraphs are either edge t-regular or not?

In the following, there are some directions which clarify the existence of some neutrosophic hypergraphs which are either edge $t$-regular or not.

Example 1.8. Two neutrosophic hypergraphs are presented such that one of them is edge $t$-regular and another isn't.

(i) : Assume Figure (7). It isn't neutrosophic edge $t$-regular hypergraph.

(ii) : Suppose Figure (2). It's neutrosophic edge 3-regular hypergraph.

Definition 1.9. (Neutrosophic vertex $t$-Regular Hypergraph).

A neutrosophic hypergraph $N H G=(V, E, \sigma, \mu)$ is called a neutrosophic vertex $t$-regular hypergraph if every neutrosophic vertex is incident to only $t$ neutrosophic hyperedges.

Example 1.10. Three neutrosophic hypergraphs are presented such that one of them is vertex $t$-regular and anothers aren't.

(i) : Consider Figure (7). It isn't neutrosophic edge $t$-regular hypergraph.

(ii) : Suppose Figure (2). It's neutrosophic edge 3-regular hypergraph but It isn't neutrosophic vertex 3-regular hypergraph.

(iii) : Assume Figure (3). It's neutrosophic vertex 2-regular hypergraph but It isn't neutrosophic edge $t$-regular hypergraph. 


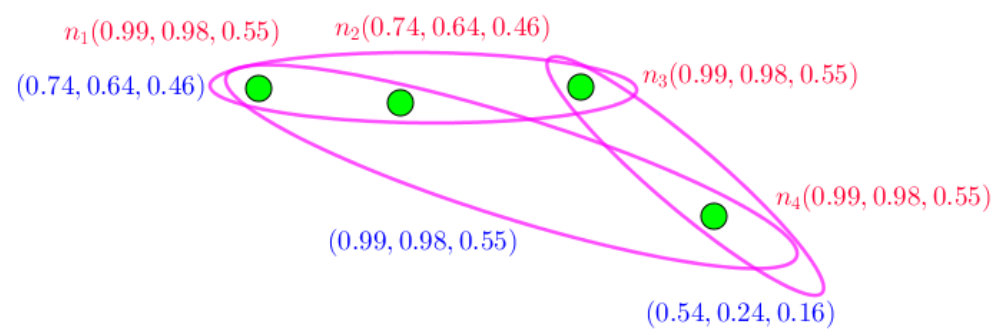

NHG

Figure 3. $N H G=(V, E, \sigma, \mu)$ is neutrosophic strong hypergraph.

Definition 1.11. (Neutrosophic Strong Hypergraph).

A neutrosophic hypergraph $N H G=(V, E, \sigma, \mu)$ is called a neutrosophic strong hypergraph if it's hypergraph and for every $v_{1} v_{2} \cdots v_{t} \in E$,

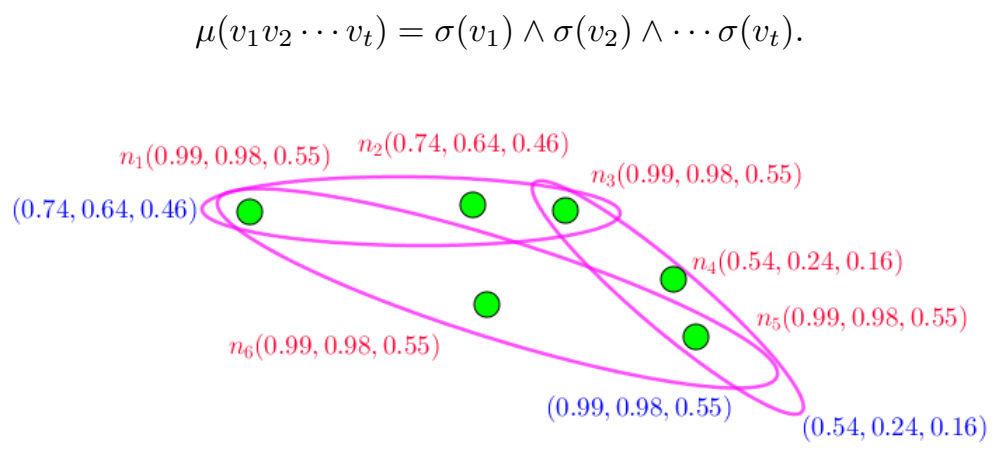

NHG

Figure 4. $N H G=(V, E, \sigma, \mu)$ is neutrosophic strong hypergraph.

Example 1.12. Three neutrosophic hypergraphs are presented such that one of them is neutrosophic strong hypergraph and others aren't.

(i) : Consider Figure (7). It isn’t neutrosophic strong hypergraph.

(ii) : Assume Figure (2). It isn't neutrosophic strong hypergraph.

(iii) : Suppose Figure (3). It isn't neutrosophic strong hypergraph.

$(i v)$ : Assume Figure (4). It's neutrosophic strong hypergraph. It's also neutrosophic edge 3 -regular hypergraph but it isn't neutrosophic vertex $t$-regular hypergraph.

Definition 1.13. (Neutrosophic Strong Hypergraph).

Assume neutrosophic hypergraph $N H G=(V, E, \sigma, \mu$.) A neutrosophic hyperedge $v_{1} v_{2} \cdots v_{t} \in E$ is called a neutrosophic strong hyperedge if

$$
\mu\left(v_{1} v_{2} \cdots v_{t}\right)=\sigma\left(v_{1}\right) \wedge \sigma\left(v_{2}\right) \wedge \cdots \sigma\left(v_{t}\right) .
$$

Proposition 1.14. Assume neutrosophic strong hypergraph $N H G=(V, E, \sigma, \mu$.) Then all neutrosophic hyperedges are neutrosophic strong.

Definition 1.15. (Neutrosophic Hyperpath).

A path $v_{0}, E_{0}, v_{1}, v_{1}, E_{1}, v_{2}, \cdots, v_{t-1}, E_{t-1}, v_{t}$, is called neutrosophic hyperpath such that $v_{i-1}$ and $v_{i}$ have incident to $E_{i-1}$ for all nonnegative integers $0 \leq i \leq t$. In this case, $t-1$ is called length of neutrosophic hyperpath. Also, if $x$ and $y$ are two 
neutrosophic vertices, then maximum length of neutrosophic hyperpaths from $x$ to $y$, is ${ }_{109}$ called neutrosophic hyperdistance and it's denoted by $d(x, y)$. If $v_{0}=v_{t}$, then it's called neutrosophic hypercycle.

Example 1.16. Assume Figure (7).

$(i): n_{1}, E_{1}, n_{3}, E_{2}, n_{6}, E_{3}, n_{1}$ is a neutrosophic hypercycle.

(ii) : $n_{1}, E_{1}, n_{n}, E_{2}, n_{6}, E_{3}, n_{1}$ isn't neither neutrosophic hypercycle nor neutrosophic hyperpath.

(iii) : $n_{1} E_{1} n_{3} E_{2} n_{6} E_{3} n_{1}$ isn't neither neutrosophic hypercycle nor neutrosophic hyperpath.

$(i v): n_{1}, n_{3}, n_{6}, n_{1}$ isn't neither neutrosophic hypercycle nor neutrosophic hyperpath.

$(v): n_{1} E_{1}, n_{3}, E_{2}, n_{6}, E_{3}, n_{1}$ isn't neither neutrosophic hypercycle nor neutrosophic hyperpath.

(vi) : $n_{1}, E_{1}, n_{3}, E_{2}, n_{6}, E_{3}, n_{7}$ is a neutrosophic hyperpath.

(vii) : Neutrosophic hyperdistance amid $n_{1}$ and $n_{4}$ is two.

(viii) : Neutrosophic hyperdistance amid $n_{1}$ and $n_{7}$ is one.

$(i x)$ : Neutrosophic hyperdistance amid $n_{1}$ and $n_{2}$ is one.

$(x)$ : Neutrosophic hyperdistance amid two given neutrosophic vertices is either one or two.

\section{New Ideas For Setting of Neutrosophic n-SuperHyperGraph}

Question 2.1. What-if the notion of complete proposes some classes of neutrosophic hypergraphs?

In the setting of neutrosophic hypergraphs, the notion of complete have introduced some classes. Since the vertex could have any number of arbitrary hyperedges. This notion is too close to the notion of regularity. Thus the idea of complete has an obvious structure in that, every hyperedge has $n$ vertices so there's only one hyperedge.

Definition 2.2. Assume neutrosophic hypergraph $N H G=(V, E, \sigma, \mu)$. It's denoted by $N H G_{n}^{r}$ and it's $(r, n)$ - regular if every hyperedge has exactly $r$ vertices in the way that, all $r$-subsets of the vertices have an unique hyperedge where $r \leq n$ and $|V|=n$.

Example 2.3. In Figure (5), $\mathrm{NHG}_{4}^{3}$ is shown.

Definition 2.4. Assume neutrosophic hypergraph $N H G=(V, E, \sigma, \mu)$.

$(i)$ : Maximum number is maximum number of hyperedges which are incident to a vertex and it's denoted by $\Delta(N H G)$;

(ii) : Minimum number is minimum number of hyperedges which are incident to a vertex and it's denoted by $\delta(N H G)$;

(iii) : Maximum value is maximum value of vertices and it's denoted by $\Delta_{n}(N H G)$;

$(i v)$ : Minimum value is minimum value of vertices and it's denoted by $\delta_{n}(N H G)$. 


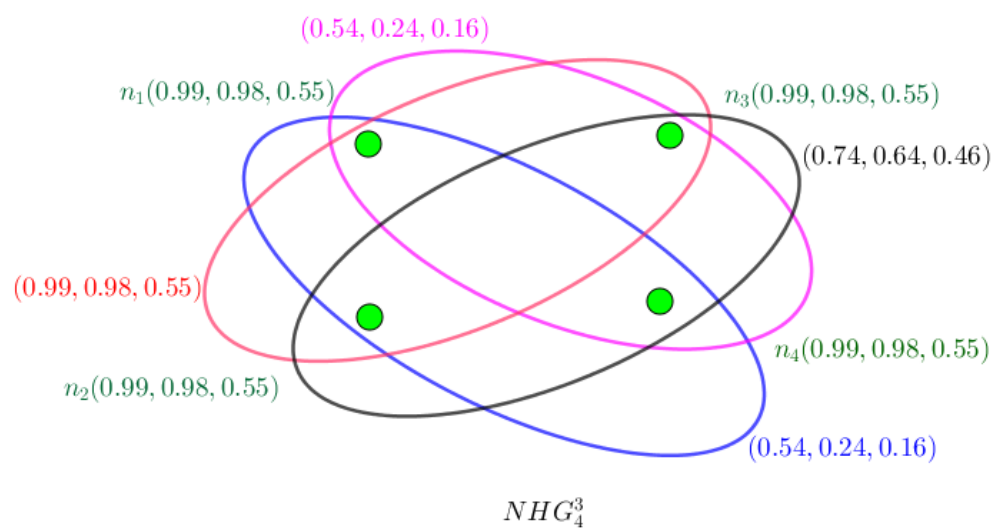

Figure 5. $N H G_{4}^{3}=(V, E, \sigma, \mu)$ is neutrosophic $(3,4)$ - regular hypergraph.

Example 2.5. Assume neutrosophic hypergraph $N H G=(V, E, \sigma, \mu)$ as Figure (5).

(i) : $\Delta(N H G)=3$;

(ii) : $\delta(N H G)=3$;

(iii) : $\Delta_{n}(N H G)=(0.99,0.98,0.55)$;

(iv) : $\delta_{n}(N H G)=(0.99,0.98,0.55)$.

Question 2.6. What-if the notion of complete proposes some classes of neutrosophic hypergraphs with some parts?

In the setting of neutrosophic hypergraphs, when every part has specific attribute inside and outside, the notion of complete is applied to parts to form the idea of completeness.

Definition 2.7. Assume neutrosophic hypergraph $N H G=(V, E, \sigma, \mu)$. It's denoted by $N H G_{n_{1}, n_{2}, \cdots, n_{r}}^{r}$ and it's complete $r$-partite if $V$ can be partitioned into $r$ non-empty parts, $V_{i}$, and every hyperedge has only one vertex from each part where $n_{i}$ is the number of vertices in part $V_{i}$.

Example 2.8. In Figure (6), $N H G_{3,3,3}^{3}=(V, E, \sigma, \mu)$ is shown.

Definition 2.9. Assume neutrosophic hypergraph $N H G=(V, E, \sigma, \mu)$.

(i) : A neutrosophic number of vertices $x_{1}, x_{2}, \cdots, x_{n}$ is

$$
\sum_{i=1}^{n} \sigma\left(x_{i}\right)
$$

(ii) : A neutrosophic number of hyperedges $e_{1}, e_{2}, \cdots, e_{n}$ is

$$
\sum_{i=1}^{n} \mu\left(e_{i}\right) .
$$

Example 2.10. I get some clarifications about new definitions.

(i) : In Figure (5), $\mathrm{NHG}_{4}^{3}$ is shown.

(a): A neutrosophic number of vertices $n_{1}, n_{2}, n_{3}$ is

$$
\Sigma_{i=1}^{3} \sigma\left(n_{i}\right)=(2.97,2.94,1.65) .
$$




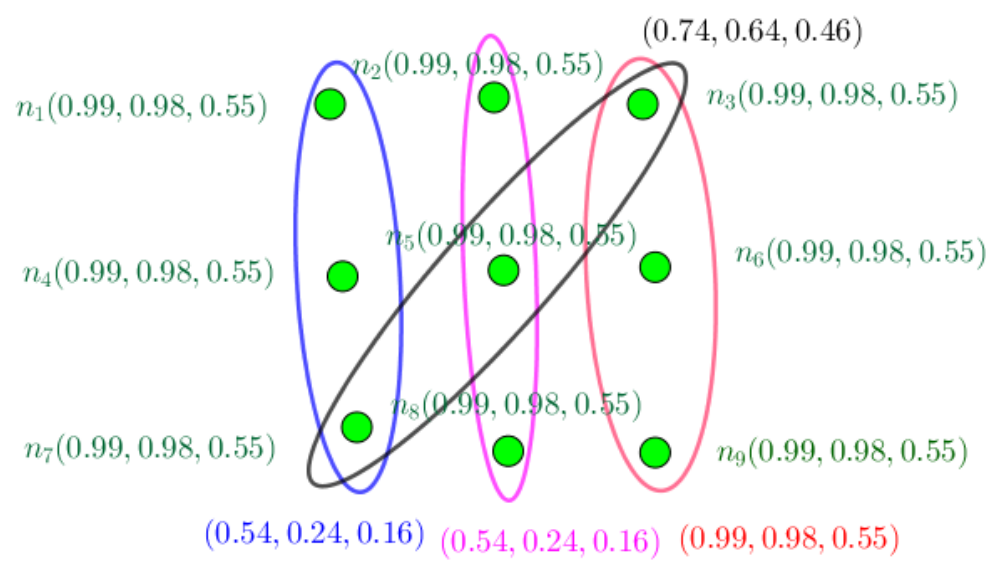

$N H G_{3,3,3}^{3}$

Figure 6. $N H G_{3,3,3}^{3}=(V, E, \sigma, \mu)$ is neutrosophic complete 3-partite hypergraph.

(b) : A neutrosophic number of hyperedges $e_{1}, e_{2}, e_{3}$ is

$$
\Sigma_{i=1}^{3} \sigma\left(e_{i}\right)=(1.82,1.12,0.78) .
$$

where $e_{1}=(0.54,0.24,0.16), e_{2}=(0.74,0.64,0.46), e_{3}=(0.54,0.24,0.16)$.

(ii) : In Figure (6), $N H G_{3,3,3}^{3}=(V, E, \sigma, \mu)$ is shown.

(a): A neutrosophic number of vertices $n_{1}, n_{2}, n_{3}$ is

$$
\Sigma_{i=1}^{3} \sigma\left(n_{i}\right)=(2.97,2.94,1.65) .
$$

(b) : A neutrosophic number of hyperedges $e_{1}, e_{2}, e_{3}$ is

$$
\Sigma_{i=1}^{3} \sigma\left(e_{i}\right)=(1.82,1.12,0.78) .
$$

where $e_{1}=(0.54,0.24,0.16), e_{2}=(0.74,0.64,0.46), e_{3}=(0.54,0.24,0.16)$.

Definition 2.11. Assume neutrosophic hypergraph $N H G=(V, E, \sigma, \mu)$.

(i) : A degree of vertex $x$ is the number of hyperedges which are incident to $x$.

(ii) : A neutrosophic degree of vertex $x$ is the neutrosophic number of hyperedges which are incident to $x$.

(iii) : A degree of hyperedge $e$ is the number of vertices which $e$ is incident to them.

(iv) : A neutrosophic degree of hyperedge $e$ is the neutrosophic number of vertices which $e$ is incident to them.

$(v)$ : A co-degree of vertices $x_{1}, x_{2}, \cdots, x_{n}$ is the number of hyperedges which are incident to $x_{1}, x_{2}, \cdots, x_{n}$.

(vi) : A neutrosophic co-degree of vertices $x_{1}, x_{2}, \cdots, x_{n}$ is the neutrosophic number of hyperedges which are incident to $x_{1}, x_{2}, \cdots, x_{n}$.

(vii) : A co-degree of hyperedges $e_{1}, e_{2}, \cdots, e_{n}$ is the number of vertices which $e_{1}, e_{2}, \cdots, e_{n}$ are incident to them. 
(viii) : A neutrosophic co-degree of hyperedges $e_{1}, e_{2}, \cdots, e_{n}$ is the neutrosophic number of vertices which $e_{1}, e_{2}, \cdots, e_{n}$ are incident to them.

Example 2.12. I get some clarifications about new definitions.

$(i)$ : In Figure (5), $N H G_{4}^{3}$ is shown.

(a) : A degree of any vertex is 3 .

(b) : A neutrosophic degree of vertex $n_{1}$ is $(2.07,1.46,0.87)$.

(c) : A degree of hyperedge $e$ where $\mu(e)=(0.99,0.98,0.55)$ is 3 .

$(d)$ : A neutrosophic degree of hyperedge $e$ where $\mu(e)=(0.99,0.98,0.55)$ is $(2.97,2.94,1.65)$.

(e) : A co-degree of vertices $n_{1}, n_{3}$ is 2 .

$(f)$ : A neutrosophic co-degree of vertices $n_{1}, n_{3}$ is $(1.53,1.22,0.71)$.

$(g)$ : A co-degree of hyperedges $e_{1}, e_{2}$ where $\mu\left(e_{1}\right)=(0.99,0.98,0.55)$ and $\mu\left(e_{2}\right)=(0.54,0.24,0.16)$ is 2 .

$(h)$ : A neutrosophic co-degree of hyperedges $e_{1}, e_{2}$ where $\mu\left(e_{1}\right)=(0.99,0.98,0.55)$ and $\mu\left(e_{2}\right)=(0.54,0.24,0.16)$ is $(1.98,1.96,1.1)$.

(ii) : In Figure (6), $N H G_{3,3,3}^{3}=(V, E, \sigma, \mu)$ is shown.

(a) : A degree of any vertex $n_{1}, n_{2}, n_{4}, n_{6}, n_{8}, n_{9}$ is 1 and degree of any vertex $n_{3}, n_{5}, n_{7}$ is 2 .

(b) : A neutrosophic degree of vertex $n_{1}, n_{2}, n_{4}, n_{6}, n_{8}, n_{9}$ is $(0.99,0.98,0.55)$ and degree of any vertex $n_{3}, n_{5}, n_{7}$ is $(1.98,1.96,1.1)$.

(c) : A degree of any hyperedge is 3 .

$(d)$ : A neutrosophic degree of hyperedge is $(2.97,2.94,1.65)$.

(e) : A co-degree of vertices $n_{1}, n_{4}$ is 1 .

$(f)$ : A neutrosophic co-degree of vertices $n_{1}, n_{4}$ is $(0.54,0.24,0.16)$.

$(g)$ : A co-degree of hyperedges $e_{1}, e_{2}$ where $\mu\left(e_{1}\right)=(0.99,0.98,0.55)$ and $\mu\left(e_{2}\right)=(0.54,0.24,0.16)$ is 1 .

(h) : A neutrosophic co-degree of hyperedges $e_{1}, e_{2}$ where $\mu\left(e_{1}\right)=(0.99,0.98,0.55)$ and $\mu\left(e_{2}\right)=(0.54,0.24,0.16)$ is $(0.99,0.98,0.55)$.

Example 2.13. Consider Figure (7) where the improvements on its super-edges to have super strong hypergraph.

(a) : The notions of dominating are clarified.

$(i): n_{1}$ super-dominates every super-vertex from the set of super-vertices $\left\{n_{7}, n_{8}, n_{9}, n_{2}, n_{3}\right\} . n_{4}$ super-dominates every super-vertex from the set of super-vertices $\left\{n_{6}, n_{5}, n_{3}\right\} . n_{4}$ doesn't super-dominate every super-vertex from the set of super-vertices $\left\{n_{1}, n_{2}, n_{7}, n_{8}, n_{9}\right\}$.

(ii) : $\left\{n_{1}, n_{3}\right\}$ is super-coloring set but $\left\{n_{1}, n_{4}\right\}$ is optimal-super-dominating set.

(iii) : $(1.53,1.22,0.71)$ is optimal-super-dominating number.

(b) : The notions of resolving are clarified.

$(i): n_{1}$ super-resolves two super-vertices $n_{4}$ and $n_{6}$.

(ii) : $V \backslash\left\{n_{1}, n_{4}\right\}$ is super-resolves set but $V \backslash\left\{n_{2}, n_{4}, n_{9}\right\}$ is optimal-super-resolving set. 
( iii $):(5,94,6.36,3.3)$ is optimal-super-resolving number.

(c) : The notions of coloring are clarified.

$(i): n_{1}$ super-colors every super-vertex from the set of super-vertices $\left\{n_{7}, n_{8}, n_{9}, n_{2}, n_{3}\right\} . n_{4}$ super-colors every super-vertex from the set of super-vertices $\left\{n_{6}, n_{5}, n_{3}\right\} . n_{4}$ doesn't super-dominate every super-vertex from the set of super-vertices $\left\{n_{1}, n_{2}, n_{7}, n_{8}, n_{9}\right\}$.

$(i i):\left\{n_{1}, n_{5}, n_{7}, n_{8}, n_{9}, n_{6}, n_{4}\right\}$ is super-coloring set but $\left\{n_{1}, n_{5}, n_{7}, n_{8}, n_{2}, n_{4}\right\}$ is optimal-super-coloring set.

(iii) : $(5.24,4.8,2.82)$ is optimal-super-coloring number.

Example 2.14. Consider Figure (3).

(a) : The notions of dominating are clarified.

$(i): n_{1}$ super-dominates every super-vertex from the set of super-vertices $\left\{n_{5}, n_{6}, n_{2}, n_{3}\right\} . n_{4}$ super-dominates every super-vertex from the set of super-vertices $\left\{n_{5}, n_{3}\right\}$. $n_{4}$ doesn't super-dominate every super-vertex from the set of super-vertices $\left\{n_{1}, n_{2}, n_{6}\right\}$.

(ii) : $\left\{n_{1}, n_{3}\right\}$ is super-dominating set but $\left\{n_{1}, n_{4}\right\}$ is optimal-super-dominating set.

(iii) : $(1.53,1.22,0.71)$ is optimal-super-dominating number.

(b) : The notions of resolving are clarified.

$(i): n_{1}$ super-resolves two super-vertices $n_{4}$ and $n_{6}$.

(ii) : $V \backslash\left\{n_{1}, n_{4}\right\}$ is super-resolves set but $V \backslash\left\{n_{2}, n_{4}, n_{6}\right\}$ is optimal-super-resolving set.

(iii) : $(5,94,6.36,3.3)$ is optimal-super-resolving number.

(c) : The notions of coloring are clarified.

$(i): n_{1}$ super-colors every super-vertex from the set of super-vertices $\left\{n_{5}, n_{6}, n_{2}, n_{3}\right\} . n_{4}$ super-colors every super-vertex from the set of super-vertices $\left\{n_{5}, n_{3}\right\}$. $n_{4}$ doesn't super-dominate every super-vertex from the set of super-vertices $\left\{n_{1}, n_{2}, n_{6}\right\}$.

(ii) : $\left\{n_{1}, n_{5}, n_{6}\right\}$ is super-coloring set but $\left\{n_{5}, n_{2}, n_{4}\right\}$ is optimal-super-coloring set.

(iii) : $(2.27,1.86,1.17)$ is optimal-super-coloring number.

\subsection{Preliminaries For Setting of n-SuperHyperGraph}

Definition 2.15. (n-SuperHyperGraph in Ref. [14]).

A graph $\left(G_{n} \subseteq P^{n}(V), E_{n} \subseteq P^{n}(V)\right)$ is called by $\mathbf{n}$-SuperHyperGraph and it's denoted by $\mathrm{n}-S H G$.

\section{New Ideas For n-SuperHyperGraph}

Definition 3.1. (Dominating, Resolving and Coloring).

Assume n-SuperHyperGraph n- $S H G=\left(G_{n} \subseteq P^{n}(V), E_{n} \subseteq P^{n}(V)\right)$.

(a) : Super-dominating set and number are defined as follows. 
( $i)$ : A super-vertex $X_{n}$ super-dominates a super-vertex $Y_{n}$ if there's at least one super-edge which have them.

(ii) : A set $S$ is called super-dominating set if for every $Y_{n} \in G_{n} \backslash S$, there's at least one super-vertex $X_{n}$ which super-dominates super-vertex $Y_{n}$.

(iii) : If $\mathcal{S}$ is set of all sets of super-dominating sets, then

$$
|X|=\min _{S \in \mathcal{S}}\left|\left\{\cup X_{n} \mid X_{n} \in S\right\}\right|
$$

is called optimal-super-dominating number and $X$ is called optimal-super-dominating set.

(b) : Super-resolving set and number are defined as follows.

(i) : A super-vertex $x$ super-resolves super-vertices $y, w$ if

$$
d(x, y) \neq d(x, w)
$$

(ii) : A set $S$ is called super-resolving set if for every $Y_{n} \in G_{n} \backslash S$, there's at least one super-vertex $X_{n}$ which super-resolves super-vertices $Y_{n}, W_{n}$.

(iii) : If $\mathcal{S}$ is set of all sets of super-resolving sets, then

$$
|X|=\min _{S \in \mathcal{S}}\left|\left\{\cup X_{n} \mid X_{n} \in S\right\}\right|
$$

is called optimal-super-resolving number and $X$ is called optimal-super-resolving set.

$(c)$ : Super-coloring set and number are defined as follows.

(i) : A super-vertex $X_{n}$ super-colors a super-vertex $Y_{n}$ differently with itself if there's at least one super-edge which is incident to them.

(ii) : A set $S_{n}$ is called super-coloring set if for every $y \in G_{n} \backslash S_{n}$, there's at least one super-vertex $X_{n}$ which super-colors super-vertex $Y_{n}$.

(iii) : If $\mathcal{S}_{n}$ is set of all sets of super-coloring sets, then

$$
|X|=\min _{S_{n} \in \mathcal{S}_{n}}\left|\left\{\cup X_{n} \mid X_{n} \in S_{n}\right\}\right|
$$

is called optimal-super-coloring number and $X$ is called optimal-super-coloring set.

\section{Optimal Numbers For n-SuperHyperGraph}

Proposition 4.1. Assume n-SuperHyperGraph

$n$-SHG $=\left(G_{n} \subseteq P^{n}(V), E_{n} \subseteq P^{n}(V)\right)$. $S$ is maximum set of super-vertices which form a super-edge. Then optimal-super-coloring set has as cardinality as $S$ has.

Proof. Assume n-SuperHyperGraph n- $S H G=\left(G_{n} \subseteq P^{n}(V), E_{n} \subseteq P^{n}(V)\right)$. Every super-edge has super-vertices which have common super-edge. Thus every super-vertex has different color with other super-vertices which are incident with a super-edge. It induces a super-edge with the most number of super-vertices determines optimal-super-coloring set. $S$ is maximum set of super-vertices which form a super-edge. Thus optimal-super-coloring set has as cardinality as $S$ has. 
Proposition 4.2. Assume n-SuperHyperGraph $n$-SHG $=\left(G_{n} \subseteq P^{n}(V), E_{n} \subseteq P^{n}(V)\right)$. If optimal-super-coloring number is

$$
|V|,
$$

then for every super-vertex there's at least one super-edge which contains has all members of $V$.

Proof. Suppose n-SuperHyperGraph n- $S H G=\left(G_{n} \subseteq P^{n}(V), E_{n} \subseteq P^{n}(V)\right)$. Consider optimal-super-coloring number is

$$
|V| \text {. }
$$

It implies there's one super-edge which has all members of $V$. Since if all members of $V$ are incident to a super-edge via a super-vertex, then all have different colors.

Proposition 4.3. Assume n-SuperHyperGraph $n$-SHG $=\left(G_{n} \subseteq P^{n}(V), E_{n} \subseteq P^{n}(V)\right)$. If there's at least one super-edge which has all members of $V$, then optimal-super-coloring number is

$$
|V| \text {. }
$$

Proof. Consider n-SuperHyperGraph n-SHG $=\left(G_{n} \subseteq P^{n}(V), E_{n} \subseteq P^{n}(V)\right)$. Suppose there's at least one super-edge which has all members of $V$. It implies there's one super-edge which has some super-vertices but all members of $V$. If all super-vertices are incident to a super-edge, then all have different colors. It means if some super-vertices have all members of $V$, in the way that, for every member of $V$, there's a distinct super-vertex which has it and all such these super-vertices are incident to a super-edge, then all have different colors. So the set of these super-vertices are $V$, is optimal-super-coloring set. It induces optimal-super-coloring number is

$$
|V| .
$$

Proposition 4.4. Assume n-SuperHyperGraph $n$-SHG $=\left(G_{n} \subseteq P^{n}(V), E_{n} \subseteq P^{n}(V)\right)$. If optimal-super-dominating number is

$$
|V|,
$$

then there's one member of $V$, is contained in, at least one super-vertex which doesn't have incident to any super-edge.

Proof. Suppose n-SuperHyperGraph n-SHG $=\left(G_{n} \subseteq P^{n}(V), E_{n} \subseteq P^{n}(V)\right)$.Consider optimal-super-dominating number is

$$
|V| \text {. }
$$

If for all given super-vertex and all members of $V$, there's at least one super-edge, which the super-vertex has incident to it, then there's a super-vertex $X_{n}$ such that optimal-super-dominating number is

$$
|V|-\left|X_{n}\right|
$$

It induces contradiction with hypothesis. It implies there's one member of $V$, is contained in, at least one super-vertex which doesn't have incident to any super-edge. 
Proposition 4.5. Assume n-SuperHyperGraph $n$-SHG $=\left(G_{n} \subseteq P^{n}(V), E_{n} \subseteq P^{n}(V)\right)$. Then optimal-super-dominating number is $<$

$$
|V| \text {. }
$$

Proof. Consider n-SuperHyperGraph n-SHG $=\left(G_{n} \subseteq P^{n}(V), E_{n} \subseteq P^{n}(V)\right)$. Thus $G_{n}-\left\{X_{n}\right\}$, is a super-dominating set where $X_{n} \in G_{n}$. Since if not, $X_{n}$ isn't incident to any given super-edge. This is contradiction with supposition. It induces that $X_{n}$ belongs to a super-edge which has another super-vertex $X_{n}^{\prime}$. It implies $X_{n}^{\prime}$ super-dominates $X_{n}$. Thus $G_{n}-\left\{X_{n}\right\}$ is a super-dominating set. It induces optimal-super-dominating number is $<$

$$
|V|
$$

Proposition 4.6. Assume n-SuperHyperGraph $n$-SHG $=\left(G_{n} \subseteq P^{n}(V), E_{n} \subseteq P^{n}(V)\right)$. If optimal-super-resolving number is

$$
|V| \text {. }
$$

then every given super-vertex doesn't have incident to any super-edge.

Proof. Consider n-SuperHyperGraph n-SHG $=\left(G_{n} \subseteq P^{n}(V), E_{n} \subseteq P^{n}(V)\right)$. Let optimal-super-resolving number be

$$
|V| \text {. }
$$

If it implies there's a super-vertex is super-resolved by a super-vertex, then it's contradiction with hypothesis. So every given super-vertex doesn't have incident to any super-edge.

Proposition 4.7. Assume n-SuperHyperGraph $n$-SHG $=\left(G_{n} \subseteq P^{n}(V), E_{n} \subseteq P^{n}(V)\right)$. Then optimal-super-resolving number is $<$

$$
|V| .
$$

Proof. Consider n-SuperHyperGraph n-SHG $=\left(G_{n} \subseteq P^{n}(V), E_{n} \subseteq P^{n}(V)\right)$. If optimal-super-resolving number is

$$
|V|,
$$

then there's a contradiction to hypothesis. Since the set $G_{n}-\left\{X_{n}\right\}$, is super-resolving set. It implies optimal-super-resolving number is $<$

$$
|V|
$$

Proposition 4.8. Assume n-SuperHyperGraph $n$-SHG $=\left(G_{n} \subseteq P^{n}(V), E_{n} \subseteq P^{n}(V)\right)$. If optimal-super-coloring number is

$$
|V|
$$

then all super-vertices which have incident to at least one super-edge. 
Proof. Suppose n-SuperHyperGraph n-SHG $=\left(G_{n} \subseteq P^{n}(V), E_{n} \subseteq P^{n}(V)\right)$. Consider optimal-super-coloring number is

$$
|V|-\left|X_{n}\right| \text {. }
$$

If for all given super-vertices, there's no super-edge which the super-vertices have incident to it, then there's super-vertex $X_{n}$ such that optimal-super-coloring number is

$$
|V|-\left|X_{n}\right| \text {. }
$$

It induces contradiction with hypothesis. It implies all super-vertices have incident to at least one super-edge.

Proposition 4.9. Assume n-SuperHyperGraph $n$-SHG $=\left(G_{n} \subseteq P^{n}(V), E_{n} \subseteq P^{n}(V)\right)$. Then optimal-super-coloring number isn't $<$

$$
|V| \text {. }
$$

Proof. Consider n-SuperHyperGraph n- $S H G=\left(G_{n} \subseteq P^{n}(V), E_{n} \subseteq P^{n}(V)\right)$. Thus $G_{n}-\left\{X_{n}\right\}$ isn't a super-coloring set. Since if not, $X_{n}$ isn't incident to any given super-edge. This is contradiction with supposition. It induces that $X_{n}$ belongs to a super-edge which has another super-vertex $S_{n}$. It implies $S_{n}$ super-colors $X_{n}$. Thus $G_{n}-\left\{X_{n}\right\}$ isn't a super-coloring set. It induces optimal-super-coloring number isn't <

$$
|V| \text {. }
$$

Proposition 4.10. Assume n-SuperHyperGraph $n$-SHG $=\left(G_{n} \subseteq P^{n}(V), E_{n} \subseteq P^{n}(V)\right)$. Then optimal-super-dominating set has cardinality which is greater than $n-1$ where $n$ is the cardinality of the set $V$.

Proof. Consider n-SuperHyperGraph n-SHG $=\left(G_{n} \subseteq P^{n}(V), E_{n} \subseteq P^{n}(V)\right)$. The set $G_{n}$ is super-dominating set. So optimal-super-dominating set has cardinality which is greater than $n-1$ where $n$ is the cardinality of the set $V$. But the set $G_{n} \backslash\left\{X_{n}\right\}$, for every given super-vertex $X_{n}$ is optimal-super-dominating set has cardinality which is greater than $n-1$ where $n$ is is the cardinality of the set $V$. The result is obtained.

Proposition 4.11. Assume n-SuperHyperGraph $n$-SHG $=\left(G_{n} \subseteq P^{n}(V), E_{n} \subseteq P^{n}(V)\right)$. $S$ is maximum set of super-vertices which form a super-edge. Then $S$ is optimal-super-coloring set and

$$
\left|\left\{\cup X_{n} \mid X_{n} \in S\right\}\right|
$$

is optimal-super-coloring number.

Proof. Suppose n-SuperHyperGraph n- $S H G=\left(G_{n} \subseteq P^{n}(V), E_{n} \subseteq P^{n}(V)\right)$. Consider $S$ is maximum set of super-vertices which form a super-edge. Thus all super-vertices of $S$ have incident to super-edge. It implies the number of different colors equals to a cardinality based on $S$. Therefore, optimal-super-coloring number $\geq$

$$
\left|\left\{\cup X_{n} \mid X_{n} \in S\right\}\right|
$$

In other hand, $S$ is maximum set of super-vertices which form a super-edge. It induces optimal-super-coloring number $\leq$

$$
\left|\left\{\cup X_{n} \mid X_{n} \in S\right\}\right|
$$

So $S$ is super-coloring set. Hence $S$ is optimal-super-coloring set and

$$
\left|\left\{\cup X_{n} \mid X_{n} \in S\right\}\right|
$$

is optimal-super-coloring number. 


\section{Optimal Sets For n-SuperHyperGraph}

Proposition 5.1. Assume n-SuperHyperGraph $n$-SHG $=\left(G_{n} \subseteq P^{n}(V), E_{n} \subseteq P^{n}(V)\right)$. If $S$ is super-dominating set, then $D$ contains $S$ is super-dominating set.

Proof. Consider n-SuperHyperGraph n-SHG $=\left(G_{n} \subseteq P^{n}(V), E_{n} \subseteq P^{n}(V)\right)$. Suppose $S$ is super-dominating set. Then all super-vertices are super-dominated. Thus $D$ contains $S$ is super-dominating set.

Proposition 5.2. Assume n-SuperHyperGraph $n$-SHG $=\left(G_{n} \subseteq P^{n}(V), E_{n} \subseteq P^{n}(V)\right)$. If $S$ is super-resolving set, then $D$ contains $S$ is super-resolving set.

Proof. Suppose n-SuperHyperGraph n- $S H G=\left(G_{n} \subseteq P^{n}(V), E_{n} \subseteq P^{n}(V)\right)$. Consider $S$ is super-resolving set. Hence All two given super-vertices are super-resolved by at least one super-vertex of $S$. It induces $D$ contains $S$ is super-resolving set.

Proposition 5.3. Assume n-SuperHyperGraph $n-S H G=\left(G_{n} \subseteq P^{n}(V), E_{n} \subseteq P^{n}(V)\right)$. If $S$ is super-coloring set, then $D$ contains $S$ is super-coloring set.

Proof. Suppose n-SuperHyperGraph n-SHG $=\left(G_{n} \subseteq P^{n}(V), E_{n} \subseteq P^{n}(V)\right)$. Consider $S$ is super-coloring set. So all super-vertices which have a common super-edge have different colors. Thus every super-vertex super-colored by a super-vertex of $S$. It induces every super-vertex which has a common super-edge has different colors with other super-vertices belong to that super-edge. Then $D$ contains $S$ is super-coloring set.

Proposition 5.4. Assume n-SuperHyperGraph $n-S H G=\left(G_{n} \subseteq P^{n}(V), E_{n} \subseteq P^{n}(V)\right)$. Then $G_{n}$ is super-dominating set.

Proof. Suppose n-SuperHyperGraph n- $S H G=\left(G_{n} \subseteq P^{n}(V), E_{n} \subseteq P^{n}(V)\right)$. Since $G_{n} \backslash\left\{X_{n}\right\}$ is super-dominating set. Then $G_{n}$ contains $G_{n} \backslash\left\{X_{n}\right\}$ is super-dominating set.

Proposition 5.5. Assume n-SuperHyperGraph $n-S H G=\left(G_{n} \subseteq P^{n}(V), E_{n} \subseteq P^{n}(V)\right)$. Then $G_{n}$ is super-resolving set.

Proof. Suppose n-SuperHyperGraph n- $S H G=\left(G_{n} \subseteq P^{n}(V), E_{n} \subseteq P^{n}(V)\right)$. If there's no super-vertex, then all super-vertices are super-resolved. Hence if I choose $G_{n}$, then there's no super-vertex such that super-vertex is super-resolved. It implies $G_{n}$ is super-resolving set but $G_{n}$ isn't optimal-super-resolving set. Since if I construct one set from $G_{n}$ such that only one super-vertex is out of $S$, then $S$ is super-resolving set. It implies $G_{n}$ isn't optimal-super-resolving set. Thus $G_{n}$ is super-resolving set.

Proposition 5.6. Assume n-SuperHyperGraph $n-S H G=\left(G_{n} \subseteq P^{n}(V), E_{n} \subseteq P^{n}(V)\right)$. Then $G_{n}$ is super-coloring set.

Proof. Suppose n-SuperHyperGraph n-SHG $=\left(G_{n} \subseteq P^{n}(V), E_{n} \subseteq P^{n}(V)\right)$. All super-vertices belong to a super-edge have to color differently. If $G_{n}$ is chosen, then all super-vertices have different colors. It induces that $t$ colors are used where $t$ is the number of super-vertices. Every super-vertex has unique color. Thus $G_{n}$ is super-coloring set. 


\section{Optimal Sets and Numbers For Family of n-SuperHyperGraph}

Proposition 6.1. Assume $\mathcal{G}$ is a family of n-SuperHyperGraph. Then $G_{n}$ is super-dominating set for all members of $\mathcal{G}$, simultaneously.

Proof. Suppose $\mathcal{G}$ is a family of n-SuperHyperGraph. Thus $G_{n}$ is super-dominating set for every given n-SuperHyperGraph of $\mathcal{G}$. It implies $G_{n}$ is super-dominating set for all members of $\mathcal{G}$, simultaneously.

Proposition 6.2. Assume $\mathcal{G}$ is a family of n-SuperHyperGraph. Then $G_{n}$ is super-resolving set for all members of $\mathcal{G}$, simultaneously.

Proof. Suppose $\mathcal{G}$ is a family of n-SuperHyperGraph. Thus $G_{n}$ is super-resolving set for every given n-SuperHyperGraph of $\mathcal{G}$. It implies $G_{n}$ is super-resolving set for all members of $\mathcal{G}$, simultaneously.

Proposition 6.3. Assume $\mathcal{G}$ is a family of n-SuperHyperGraph. Then $G_{n}$ is super-coloring set for all members of $\mathcal{G}$, simultaneously.

Proof. Suppose $\mathcal{G}$ is a family of n-SuperHyperGraph. Thus $G_{n}$ is super-coloring set for every given n-SuperHyperGraph of $\mathcal{G}$. It implies $G_{n}$ is super-coloring set for all members of $\mathcal{G}$, simultaneously.

Proposition 6.4. Assume $\mathcal{G}$ is a family of n-SuperHyperGraph. Then $G_{n} \backslash\left\{X_{n}\right\}$ is super-dominating set for all members of $\mathcal{G}$, simultaneously.

Proof. Suppose $\mathcal{G}$ is a family of n-SuperHyperGraph. Thus $G_{n} \backslash\left\{X_{n}\right\}$ is super-dominating set for every given n-SuperHyperGraph of $\mathcal{G}$. One super-vertex is out of $G_{n} \backslash\left\{X_{n}\right\}$. It's super-dominated from any super-vertex in $G_{n} \backslash\left\{X_{n}\right\}$. Hence every given two super-vertices are super-dominated from any super-vertex in $G_{n} \backslash\left\{X_{n}\right\}$. It implies $G_{n} \backslash\left\{X_{n}\right\}$ is super-dominating set for all members of $\mathcal{G}$, simultaneously.

Proposition 6.5. Assume $\mathcal{G}$ is a family of n-SuperHyperGraph. Then $G_{n} \backslash\left\{X_{n}\right\}$ is super-resolving set for all members of $\mathcal{G}$, simultaneously.

Proof. Suppose $\mathcal{G}$ is a family of n-SuperHyperGraph. Thus $G_{n} \backslash\left\{X_{n}\right\}$ is super-resolving set for every given n-SuperHyperGraph of $\mathcal{G}$. One super-vertex is out of $G_{n} \backslash\left\{X_{n}\right\}$. It's super-resolved from any super-vertex in $G_{n} \backslash\left\{X_{n}\right\}$. Hence every given two super-vertices are super-resolved from any super-vertex in $G_{n} \backslash\left\{X_{n}\right\}$. It implies $G_{n} \backslash\left\{X_{n}\right\}$ is super-resolving set for all members of $\mathcal{G}$, simultaneously.

Proposition 6.6. Assume $\mathcal{G}$ is a family of n-SuperHyperGraph. Then $G_{n} \backslash\left\{X_{n}\right\}$ isn't super-coloring set for all members of $\mathcal{G}$, simultaneously.

Proof. Suppose $\mathcal{G}$ is a family of n-SuperHyperGraph. Thus $G_{n} \backslash\left\{X_{n}\right\}$ isn't super-coloring set for every given n-SuperHyperGraph of $\mathcal{G}$. One super-vertex is out of $G_{n} \backslash\left\{X_{n}\right\}$. It isn't super-colored from any super-vertex in $G_{n} \backslash\left\{X_{n}\right\}$. Hence every given two super-vertices aren't super-colored from any super-vertex in $G_{n} \backslash\left\{X_{n}\right\}$. It implies $G_{n} \backslash\left\{X_{n}\right\}$ isn't super-coloring set for all members of $\mathcal{G}$, simultaneously.

Proposition 6.7. Assume $\mathcal{G}$ is a family of $n$-SuperHyperGraph. Then union of super-dominating sets from each member of $\mathcal{G}$ is super-dominating set for all members of $\mathcal{G}$, simultaneously. 
Proof. Suppose $\mathcal{G}$ is a family of n-SuperHyperGraph. For every chosen n-SuperHyperGraph, there's one super-dominating set in the union of super-dominating sets from each member of $\mathcal{G}$. Thus union of super-dominating sets from each member of $\mathcal{G}$ is super-dominating set for every given n-SuperHyperGraph of $\mathcal{G}$. Even one super-vertex isn't out of the union. It's super-dominated from any super-vertex in the union. Hence every given two super-vertices are super-dominated from any super-vertex in union of super-coloring sets. It implies union of super-coloring sets is super-dominating set for all members of $\mathcal{G}$, simultaneously.

Proposition 6.8. Assume $\mathcal{G}$ is a family of n-SuperHyperGraph. Then union of super-resolving sets from each member of $\mathcal{G}$ is super-resolving set for all members of $\mathcal{G}$, simultaneously.

Proof. Suppose $\mathcal{G}$ is a family of n-SuperHyperGraph. For every chosen n-SuperHyperGraph, there's one super-resolving set in the union of super-resolving sets from each member of $\mathcal{G}$. Thus union of super-resolving sets from each member of $\mathcal{G}$ is super-resolving set for every given n-SuperHyperGraph of $\mathcal{G}$. Even one super-vertex isn't out of the union. It's super-resolved from any super-vertex in the union. Hence every given two super-vertices are super-resolved from any super-vertex in union of super-coloring sets. It implies union of super-coloring sets is super-resolved set for all members of $\mathcal{G}$, simultaneously.

Proposition 6.9. Assume $\mathcal{G}$ is a family of n-SuperHyperGraph. Then union of super-coloring sets from each member of $\mathcal{G}$ is super-coloring set for all members of $\mathcal{G}$, simultaneously.

Proof. Suppose $\mathcal{G}$ is a family of n-SuperHyperGraph. For every chosen n-SuperHyperGraph, there's one super-coloring set in the union of super-coloring sets from each member of $\mathcal{G}$. Thus union of super-coloring sets from each member of $\mathcal{G}$ is super-coloring set for every given n-SuperHyperGraph of $\mathcal{G}$. Even one super-vertex isn't out of the union. It's super-colored from any super-vertex in the union. Hence every given two super-vertices are super-colored from any super-vertex in union of super-coloring sets. It implies union of super-coloring sets is super-colored set for all members of $\mathcal{G}$, simultaneously.

Proposition 6.10. Assume $\mathcal{G}$ is a family of $n$-SuperHyperGraph. For every given super-vertex, there's one n-SuperHyperGraph such that the super-vertex has another super-vertex which are incident to a super-edge. If for given super-vertex, all super-vertices have a common super-edge in this way, then $G_{n} \backslash\left\{X_{n}\right\}$ is optimal-super-dominating set for all members of $\mathcal{G}$, simultaneously.

Proof. Suppose $\mathcal{G}$ is a family of n-SuperHyperGraph. For all n-SuperHyperGraph, there's no super-dominating set from any of member of $\mathcal{G}$. Thus $G_{n} \backslash\left\{X_{n}\right\}$ is super-dominating set for every given n-SuperHyperGraph of $\mathcal{G}$. For every given super-vertex, there's one n-SuperHyperGraph such that the super-vertex has another super-vertex which are incident to a super-edge. Only one super-vertex is out of $V \backslash\{x\}$. It's super-dominated from any super-vertex in the $V \backslash\{x\}$. Hence every given two super-vertices are super-dominated from any super-vertex in $G_{n} \backslash\left\{X_{n}\right\}$ It implies $G_{n} \backslash\left\{X_{n}\right\}$ is super-dominating set for all members of $\mathcal{G}$, simultaneously. If for given super-vertex, all super-vertices have a common super-edge in this way, then $G_{n} \backslash\left\{X_{n}\right\}$ is optimal-super-dominating set for all members of $\mathcal{G}$, simultaneously.

Proposition 6.11. Assume $\mathcal{G}$ is a family of $n$-SuperHyperGraph. For every given super-vertex, there's one n-SuperHyperGraph such that the super-vertex has another super-vertex which are incident to a super-edge. If for given super-vertex, all 
super-vertices have a common super-edge in this way, then $G_{n} \backslash\left\{X_{n}\right\}$ is optimal-super-resolving set for all members of $\mathcal{G}$, simultaneously.

Proof. Suppose $\mathcal{G}$ is a family of n-SuperHyperGraph. For all n-SuperHyperGraph, there's no super-resolving set from any of member of $\mathcal{G}$. Thus $G_{n} \backslash\left\{X_{n}\right\}$ is super-resolving set for every given n-SuperHyperGraph of $\mathcal{G}$. For every given super-vertex, there's one n-SuperHyperGraph such that the super-vertex has another super-vertex which are incident to a super-edge. Only one super-vertex is out of $G_{n} \backslash\left\{X_{n}\right\}$. It's super-resolved from any super-vertex in the $G_{n} \backslash\left\{X_{n}\right\}$. Hence every given two super-vertices are super-resolving from any super-vertex in $G_{n} \backslash\left\{X_{n}\right\}$. It implies $G_{n} \backslash\left\{X_{n}\right\}$ is super-resolved set for all members of $\mathcal{G}$, simultaneously. If for given super-vertex, all super-vertices have a common super-edge in this way, then $G_{n} \backslash\left\{X_{n}\right\}$ is optimal-super-resolving set for all members of $\mathcal{G}$, simultaneously.

Proposition 6.12. Assume $\mathcal{G}$ is a family of n-SuperHyperGraph. For every given super-vertex, there's one n-SuperHyperGraph such that the super-vertex has another super-vertex which are incident to a super-edge. If for given super-vertex, all super-vertices have a common super-edge in this way, then $G_{n}$ is optimal-super-coloring set for all members of $\mathcal{G}$, simultaneously.

Proof. Suppose $\mathcal{G}$ is a family of n-SuperHyperGraph. For all n-SuperHyperGraph, there's no super-coloring set from any of member of $\mathcal{G}$. Thus $G_{n}$ is super-coloring set for every given n-SuperHyperGraph of $\mathcal{G}$. For every given super-vertex, there's one n-SuperHyperGraph such that the super-vertex has another super-vertex which are incident to a super-edge. No super-vertex is out of $G_{n}$. It's super-colored from any super-vertex in the $G_{n}$. Hence every given two super-vertices are super-colored from any super-vertex in $G_{n}$. It implies $G_{n}$ is super-coloring set for all members of $\mathcal{G}$,

simultaneously. If for given super-vertex, all super-vertices have a common super-edge in this way, then $G_{n}$ is optimal-super-coloring set for all members of $\mathcal{G}$, simultaneously.

\section{Twin Super-vertices in n-SuperHyperGraph}

Proposition 7.1. Let $n$-SHG be a n-SuperHyperGraph. An $(k-1)$-set from an $k$-set of twin super-vertices is subset of a super-resolving set.

Proof. If $X_{n}$ and $X_{n}^{\prime}$ are twin super-vertices, then $N\left(X_{n}\right)=N\left(X_{n}^{\prime}\right)$. It implies $d\left(X_{n}, T_{n}\right)=d\left(X_{n}^{\prime}, T_{n}\right)$ for all $T_{n} \in G_{n}$.

Corollary 7.2. Let $n$-SHG be a n-SuperHyperGraph. The number of twin super-vertices is $n-1$. Then super-resolving number is $n-2$.

Proof. Let $X_{n}$ and $X_{n}^{\prime}$ be two super-vertices. By supposition, the cardinality of set of twin super-vertices is $n-2$. Thus there are two cases. If both are twin super-vertices, then $N\left(X_{n}\right)=N\left(X_{n}^{\prime}\right)$. It implies $d\left(X_{n}, T_{n}\right)=d\left(X_{n}^{\prime}, T_{n}\right)$ for all $T_{n} \in G_{n}$. Thus suppose if not, then let $X_{n}$ be a super-vertex which isn't twin super-vertices with any given super-vertex and let $X_{n}^{\prime}$ be a super-vertex which is twin super-vertices with any given super-vertex but not $X_{n}$. By supposition, it's possible and this is only case. Therefore, any given distinct super-vertex super-resolves $X_{n}$ and $X_{n}^{\prime}$. Then $G_{n} \backslash\left\{X_{n}, X_{n}^{\prime}\right\}$ is super-resolving set. It implies -super-resolving number is $n-2$.

Corollary 7.3. Let $n$-SHG be n-SuperHyperGraph. The number of twin super-vertices is $n-1$. Then super-resolving number is $n-2$. Every $(n-2)$-set including twin super-vertices is super-resolving set. 
Proof. By Corollary (7.2), super-resolving number is $n-2$. By n- $S H G$ is n-SuperHyperGraph, one super-vertex doesn't belong to set of twin super-vertices and a vertex from that set, are out of super-resolving set. It induces every $(n-2)$-set including twin super-vertices is super-resolving set.

Proposition 7.4. Let $n$-SHG be n-SuperHyperGraph such that it's complete. Then super-resolving number is $n-1$. Every $(n-1)$-set is super-resolving set.

Proof. In complete, every couple of super-vertices are twin super-vertices. By n- $S H G$ is complete, every couple of super-vertices are twin super-vertices. Thus by Proposition (7.1), the result follows.

Proposition 7.5. Let $\mathcal{G}$ be a family of $n$-SuperHyperGraphs with common super vertex set $G_{n}$. Then simultaneously super-resolving number of $\mathcal{G}$ is $|V|-1$

Proof. Consider $(|V|-1)$-set. Thus there's no couple of super-vertices to be super-resolved. Therefore, every $(|V|-1)$-set is super-resolving set for any given n-SuperHyperGraph. Then it holds for any n-SuperHyperGraph. It implies it's super-resolving set and its cardinality is super-resolving number. $(|V|-1)$-set has the cardinality $|V|-1$. Then it holds for any n-SuperHyperGraph. It induces it's simultaneously super-resolving set and its cardinality is simultaneously super-resolving number.

Proposition 7.6. Let $\mathcal{G}$ be a family of $n$-SuperHyperGraphs with common super-vertex set $G_{n}$. Then simultaneously super-resolving number of $\mathcal{G}$ is greater than the maximum super-resolving number of $n-S H G \in \mathcal{G}$.

Proof. Suppose $t$ and $t^{\prime}$ are simultaneously super-resolving number of $\mathcal{G}$ and super-resolving number of $\mathrm{n}-S H G \in \mathcal{G}$. Thus $t$ is super-resolving number for any $\mathrm{n}-S H G \in \mathcal{G}$. Hence, $t \geq t^{\prime}$. So simultaneously super-resolving number of $\mathcal{G}$ is greater

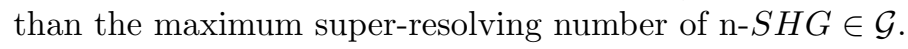

Proposition 7.7. Let $\mathcal{G}$ be a family of $n$-SuperHyperGraphs with common super-vertex set $G_{n}$. Then simultaneously super-resolving number of $\mathcal{G}$ is greater than simultaneously super-resolving number of $\mathcal{H} \subseteq \mathcal{G}$.

Proof. Suppose $t$ and $t^{\prime}$ are simultaneously super-resolving number of $\mathcal{G}$ and $\mathcal{H}$. Thus $t$

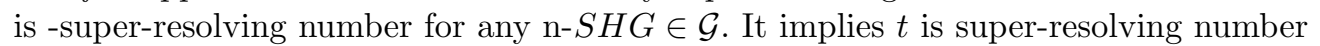
for any $n-S H G \in \mathcal{H}$. So $t$ is simultaneously super-resolving number of $\mathcal{H}$. By applying Definition about being the minimum number, $t \geq t^{\prime}$. So simultaneously super-resolving number of $\mathcal{G}$ is greater than simultaneously super-resolving number of $\mathcal{H} \subseteq \mathcal{G}$.

Theorem 7.8. Twin super-vertices aren't super-resolved in any given n-SuperHyperGraph.

Proof. Let $X_{n}$ and $X_{n}^{\prime}$ be twin super-vertices. Then $N\left(X_{n}\right)=N\left(X_{n}^{\prime}\right)$. Thus for every given super-vertex $S_{n}^{\prime} \in G_{n}, d_{\mathrm{n}-S H G}\left(s^{\prime}, t\right)=d_{\mathrm{n}-S H G}(s, t)$ where n-SHG is a given $\mathrm{n}$-SuperHyperGraph. It means that $t$ and $t^{\prime}$ aren't super-resolved in any given n-SuperHyperGraph. $t$ and $t^{\prime}$ are arbitrary so twin super-vertices aren't super-resolved in any given n-SuperHyperGraph.

Proposition 7.9. Let $n-S H G=\left(G_{n} \subseteq P^{n}(V), E_{n} \subseteq P^{n}(V)\right)$ be a $n$-SuperHyperGraph. If n-SuperHyperGraph $n$-SHG $=\left(G_{n} \subseteq P^{n}(V), E_{n} \subseteq P^{n}(V)\right)$ is complete, then every couple of super-vertices are twin super-vertices.

Proof. Let $X_{n}$ and $X_{n}^{\prime}$ be couple of given super-vertices. By n-SHG is complete, $N\left(X_{n}\right)=N\left(X_{n}^{\prime}\right)$. Thus $X_{n}$ and $X_{n}^{\prime}$ are twin super-vertices. $X_{n}$ and $X_{n}^{\prime}$ are arbitrary couple of super-vertices, hence every couple of super-vertices are twin super-vertices. 
Theorem 7.10. Let $\mathcal{G}$ be a family of n-SuperHyperGraphs $n$-SHG $=\left(G_{n} \subseteq P^{n}(V), E_{n} \subseteq P^{n}(V)\right)$ with super-vertex set $G_{n}$ and $n-S H G \in \mathcal{G}$ is complete. Then simultaneously super-resolving number is $|V|-1$. Every $(n-1)$-set is simultaneously super-resolving set for $\mathcal{G}$.

Proof. Suppose n- $S H G \in \mathcal{G}$ is SuperHyperGraph and it's complete. So by Theorem (7.9), I get every couple of super-vertices in complete SuperHyperGraph are twin super-vertices. So every couple of super-vertices, by Theorem (7.8), aren't super-resolved.

Corollary 7.11. Let $\mathcal{G}$ be a family of n-SuperHyperGraphs $n-S H G=\left(G_{n} \subseteq P^{n}(V), E_{n} \subseteq P^{n}(V)\right)$ with super-vertex set $G_{n}$ and $n-S H G \in \mathcal{G}$ is complete. Then simultaneously super-resolving number is $|V|-1$. Every $(|V|-1)$-set is simultaneously super-resolving set for $\mathcal{G}$.

Proof. It's complete. So by Theorem (7.10), I get intended result.

Theorem 7.12. Let $\mathcal{G}$ be a family of n-SuperHyperGraphs $n-S H G=\left(G_{n} \subseteq P^{n}(V), E_{n} \subseteq P^{n}(V)\right)$ with super-vertex set $G_{n}$ and for every given couple of super-vertices, there's a $n-S H G \in \mathcal{G}$ such that in that, they're twin super-vertices. Then simultaneously super-resolving number is $|V|-1$. Every $(|V|-1)$-set is simultaneously super-resolving set for $\mathcal{G}$.

Proof. By Proposition (7.5), simultaneously super-resolving number is $|V|-1$. Also, every $(|V|-1)$-set is simultaneously super-resolving set for $\mathcal{G}$.

Theorem 7.13. Let $\mathcal{G}$ be a family of n-SuperHyperGraphs $n$-SHG $=\left(G_{n} \subseteq P^{n}(V), E_{n} \subseteq P^{n}(V)\right)$ with super-vertex set $G_{n}$. If $\mathcal{G}$ contains three super-stars with different super-centers, then simultaneously super-resolving number is $|V|-2$. Every $(|V|-2)$-set is simultaneously super-resolving set for $\mathcal{G}$.

Proof. The cardinality of set of twin super-vertices is $|V|-1$. Thus by Corollary (7.3), the result follows.

Corollary 7.14. Let $\mathcal{G}$ be a family of n-SuperHyperGraphs $n-S H G=\left(G_{n} \subseteq P^{n}(V), E_{n} \subseteq P^{n}(V)\right)$ with super-vertex set $G_{n}$. If $\mathcal{G}$ contains three super-stars with different super-centers, then simultaneously super-resolving number is $|V|-2$. Every $(|V|-2)$-set is simultaneously super-resolving set for $\mathcal{G}$.

Proof. $\mathcal{G}$ be a family of n-SuperHyperGraphs n-SHG $=\left(G_{n} \subseteq P^{n}(V), E_{n} \subseteq P^{n}(V)\right)$ with super-vertex set $G_{n}$. It's complete. So by Theorem (7.13), I get intended result.

\section{Antipodal super-vertices in n-SuperHyperGraph}

\subsection{Even super-cycle}

Proposition 8.1. Consider two antipodal super-vertices $X_{n}$ and $Y_{n}$ in any given even super-cycle. Let $U_{n}$ and $V_{n}$ be given super-vertices. Then $d\left(X_{n}, U_{n}\right) \neq d\left(X_{n}, V_{n}\right)$ if and only if $d\left(Y_{n}, U_{n}\right) \neq d\left(Y_{n}, V_{n}\right)$.

Proof. $(\Rightarrow)$. Consider $d\left(X_{n}, U_{n}\right) \neq d\left(X_{n}, V_{n}\right)$. By $d\left(X_{n}, U_{n}\right)+d\left(U_{n}, Y_{n}\right)=d\left(X_{n}, Y_{n}\right)=$ $D(\mathrm{n}-S H G), D(\mathrm{n}-S H G)-d\left(X_{n}, U_{n}\right) \neq D(\mathrm{n}-S H G)-d\left(X_{n}, V_{n}\right)$. It implies $d\left(Y_{n}, U_{n}\right) \neq d\left(Y_{n}, V_{n}\right)$.

$(\Leftarrow)$. Consider $d\left(Y_{n}, U_{n}\right) \neq d\left(Y_{n}, V_{n}\right)$. By $d\left(Y_{n}, U_{n}\right)+d\left(U_{n}, X_{n}\right)=d\left(X_{n}, Y_{n}\right)=$ $D(\mathrm{n}-S H G), D(\mathrm{n}-S H G)-d\left(Y_{n}, U_{n}\right) \neq D(\mathrm{n}-S H G)-d\left(Y_{n}, V_{n}\right)$. It implies $d\left(X_{n}, U_{n}\right) \neq d\left(X_{n}, V_{n}\right)$. 
Proposition 8.2. Consider two antipodal super-vertices $X_{n}$ and $Y_{n}$ in any given even cycle. Let $U_{n}$ and $V_{n}$ be given super-vertices. Then $d\left(X_{n}, U_{n}\right)=d\left(X_{n}, V_{n}\right)$ if and only if $d\left(Y_{n}, U_{n}\right)=d\left(Y_{n}, V_{n}\right)$.

Proof. $(\Rightarrow)$. Consider $d\left(X_{n}, U_{n}\right)=d\left(X_{n}, V_{n}\right)$. By $d\left(X_{n}, U_{n}\right)+d\left(U_{n}, Y_{n}\right)=d\left(X_{n}, Y_{n}\right)=$ $D(\mathrm{n}-S H G), D(\mathrm{n}-S H G)-d\left(X_{n}, U_{n}\right)=D(\mathrm{n}-S H G)-d\left(X_{n}, V_{n}\right)$. It implies $d\left(Y_{n}, U_{n}\right)=d\left(Y_{n}, V_{n}\right)$.

$(\Leftarrow)$. Consider $d\left(Y_{n}, U_{n}\right)=d\left(Y_{n}, V_{n}\right)$. By $d\left(Y_{n}, U_{n}\right)+d\left(U_{n}, X_{n}\right)=d\left(X_{n}, Y_{n}\right)=$ $D(\mathrm{n}-S H G), D(\mathrm{n}-S H G)-d\left(Y_{n}, U_{n}\right)=D(\mathrm{n}-S H G)-d\left(Y_{n}, V_{n}\right)$. It implies $d\left(X_{n}, U_{n}\right)=d\left(X_{n}, V_{n}\right)$.

Proposition 8.3. The set contains two antipodal super-vertices, isn't super-resolving set in any given even super-cycle.

Proof. Let $X_{n}$ and $Y_{n}$ be two given antipodal super-vertices in any given even super-cycle. By Proposition (8.1), $d\left(X_{n}, U_{n}\right) \neq d\left(X_{n}, V_{n}\right)$ if and only if $d\left(Y_{n}, U_{n}\right) \neq d\left(Y_{n}, V_{n}\right)$. It implies that if $X_{n}$ super-resolves a couple of super-vertices, then $Y_{n}$ super-resolves them, too. Thus either $X_{n}$ is in a super-resolving set or $Y_{n}$ is in. It induces the set contains two antipodal super-vertices, isn't super-resolving set in any given even super-cycle.

Proposition 8.4. Consider two antipodal super-vertices $X_{n}$ and $Y_{n}$ in any given even super-cycle. $X_{n}$ super-resolves a given couple of super-vertices, $Z_{n}$ and $Z_{n}^{\prime}$, if and only if $Y_{n}$ does.

Proof. $(\Rightarrow) . X_{n}$ super-resolves a given couple of super-vertices, $Z_{n}$ and $Z_{n}^{\prime}$, then $d\left(X_{n}, Z_{n}\right) \neq d\left(X_{n}, Z_{n}^{\prime}\right)$. By Proposition (8.1), $d\left(X_{n}, Z_{n}\right) \neq d\left(X_{n}, Z_{n}^{\prime}\right)$ if and only if $d\left(Y_{n}, Z_{n}\right) \neq d\left(Y_{n}, Z_{n}^{\prime}\right)$. Thus $Y_{n}$ super-resolves a given couple of super-vertices $Z_{n}$ and $Z_{n}^{\prime}$.

$(\Leftarrow) . Y_{n}$ super-resolves a given couple of super-vertices, $Z_{n}$ and $Z_{n}^{\prime}$, then $d\left(Y_{n}, Z_{n}\right) \neq d\left(Y_{n}, Z_{n}^{\prime}\right)$. By Proposition (8.1), $d\left(Y_{n}, Z_{n}\right) \neq d\left(Y_{n}, Z_{n}^{\prime}\right)$ if and only if $d\left(X_{n}, Z_{n}\right) \neq d\left(X_{n}, Z_{n}^{\prime}\right)$. Thus $X_{n}$ super-resolves a given couple of super-vertices $Z_{n}$ and $Z_{n}^{\prime}$.

Proposition 8.5. There are two antipodal super-vertices aren't super-resolved by other two antipodal super-vertices in any given even super-cycle.

Proof. Suppose $X_{n}$ and $Y_{n}$ are a couple of super-vertices. It implies $d\left(X_{n}, Y_{n}\right)=D(\mathrm{n}-S H G)$. Consider $U_{n}$ and $V_{n}$ are another couple of super-vertices such that $d\left(X_{n}, U_{n}\right)=\frac{D(\mathrm{n}-S H G)}{2}$. It implies $d\left(Y_{n}, U_{n}\right)=\frac{D(\mathrm{n}-S H G)}{2}$. Thus $d\left(X_{n}, U_{n}\right)=d\left(Y_{n}, U_{n}\right)$. Therefore, $U_{n}$ doesn't super-resolve a given couple of super-vertices $X_{n}$ and $Y_{n}$. By

$D(\mathrm{n}-S H G G)=d\left(U_{n}, V_{n}\right)=d\left(U_{n}, X_{n}\right)+d\left(X_{n}, V_{n}\right)=\frac{D(\mathrm{n}-S H G)}{2}+d\left(X_{n}, V_{n}\right)$, $d\left(X_{n}, V_{n}\right)=\frac{D(\mathrm{n}-S H G)}{2}$. It implies $d\left(Y_{n}, V_{n}\right)=\frac{D(\mathrm{n}-S H G)}{2}$. Thus $d\left(X_{n}, V_{n}\right)=d\left(Y_{n}, V_{n}\right)$. Therefore, $V_{n}$ doesn't super-resolve a given couple of super-vertices $X_{n}$ and $Y_{n}$.

Proposition 8.6. For any two antipodal super-vertices in any given even super-cycle, there are only two antipodal super-vertices don't super-resolve them.

Proof. Suppose $X_{n}$ and $Y_{n}$ are a couple of super-vertices such that they're antipodal super-vertices. Let $U_{n}$ be a super-vertex such that $d\left(X_{n}, U_{n}\right)=\frac{D(\mathrm{n}-S H G)}{2}$. It implies $d\left(Y_{n}, U_{n}\right)=\frac{D(\mathrm{n}-S H G)}{2}$. Thus $d\left(X_{n}, U_{n}\right)=d\left(Y_{n}, U_{n}\right)$. Therefore, $U_{n}$ doesn't super-resolve a given couple of super-vertices $X_{n}$ and $Y_{n}$. Let $V_{n}$ be a antipodal vertex for $U_{n}$ such that $U_{n}$ and $V_{n}$ are antipodal super-vertices. Thus $V_{n}$

$d\left(X_{n}, V_{n}\right)=\frac{D(\mathrm{n}-S H G)}{2}$. It implies $d\left(Y_{n}, V_{n}\right)=\frac{D(\mathrm{n}-S H G)}{2}$. Therefore, $V_{n}$ doesn't 
super-resolve a given couple of super-vertices $X_{n}$ and $Y_{n}$. If $U_{n}$ is a super-vertex such that $d\left(X_{n}, U_{n}\right) \neq \frac{D(\mathrm{n}-S H G)}{2}$ and $V_{n}$ is a super-vertex such that $U_{n}$ and $V_{n}$ are antipodal super-vertices. Thus $d\left(X_{n}, V_{n}\right) \neq \frac{D(\mathrm{n}-S H G)}{2}$ It induces either $d\left(X_{n}, U_{n}\right) \neq d\left(Y_{n}, U_{n}\right)$ or $d\left(X_{n}, V_{n}\right) \neq d\left(Y_{n}, V_{n}\right)$. It means either $U_{n}$ super-resolves a given couple of super-vertices $X_{n}$ and $Y_{n}$ or $V_{n}$ super-resolves a given couple of super-vertices $X_{n}$ and $Y_{n}$.

Proposition 8.7. In any given even super-cycle, for any super-vertex, there's only one super-vertex such that they're antipodal super-vertices.

Proof. If $d\left(X_{n}, Y_{n}\right)=D(\mathrm{n}-S H G)$, then $X_{n}$ and $Y_{n}$ are antipodal super-vertices.

Proposition 8.8. Let $n$-SuperHyperGraphs $n$-SHG $=\left(G_{n} \subseteq P^{n}(V), E_{n} \subseteq P^{n}(V)\right)$ be an even super-cycle. Then every couple of super-vertices are super-resolving set if and only if they aren't antipodal super-vertices.

Proof. If $X_{n}$ and $Y_{n}$ are antipodal super-vertices, then they don't super-resolve a given couple of super-vertices $U_{n}$ and $V_{n}$ such that they're antipodal super-vertices and $d\left(X_{n}, U_{n}\right)=\frac{D(\mathrm{n}-S H G)}{2}$. Since

$d\left(X_{n}, U_{n}\right)=d\left(X_{n}, V_{n}\right)=d\left(Y_{n}, u\right)=d\left(Y_{n}, V_{n}\right)=\frac{D(\mathrm{n}-S H G)}{2}$.

Corollary 8.9. Let $n$-SuperHyperGraphs $n$-SHG $=\left(G_{n} \subseteq P^{n}(V), E_{n} \subseteq P^{n}(V)\right)$ be an even super-cycle. Then super-resolving number is two.

Proof. A set contains one super-vertex $X_{n}$ isn't super-resolving set. Since it doesn't super-resolve a given couple of super-vertices $U_{n}$ and $V_{n}$ such that

$d\left(X_{n}, U_{n}\right)=d\left(X_{n}, V_{n}\right)=1$. Thus super-resolving number $\geq 2$. By Proposition (8.8), every couple of super-vertices such that they aren't antipodal super-vertices, are

super-resolving set. Therefore, super-resolving number is 2 .

Corollary 8.10. Let n-SuperHyperGraphs $n$-SHG $=\left(G_{n} \subseteq P^{n}(V), E_{n} \subseteq P^{n}(V)\right)$ be an even super-cycle. Then super-resolving set contains couple of super-vertices such that they aren't antipodal super-vertices.

Proof. By Corollary (8.9), super-resolving number is two. By Proposition (8.8), every couple of super-vertices such that they aren't antipodal super-vertices, form

super-resolving set. Therefore, super-resolving set contains couple of super-vertices such that they aren't antipodal super-vertices.

Corollary 8.11. Let $\mathcal{G}$ be a family n-SuperHyperGraphs

$n$-SHG $=\left(G_{n} \subseteq P^{n}(V), E_{n} \subseteq P^{n}(V)\right)$ be an odd super-cycle with common super-vertex set $G_{n}$. Then simultaneously super-resolving set contains couple of super-vertices such that they aren't antipodal super-vertices and super-resolving number is two.

\subsection{Odd super-cycle}

Proposition 8.12. In any given n-SuperHyperGraph $n$-SHG $=\left(G_{n} \subseteq P^{n}(V), E_{n} \subseteq P^{n}(V)\right)$ which is odd super-cycle, for any super-vertex, there's no super-vertex such that they're antipodal super-vertices.

Proof. if $X_{n}$ is a given super-vertex. Then there are two super-vertices $U_{n}$ and $V_{n}$ such that $d\left(X_{n}, U_{n}\right)=d\left(X_{n}, V_{n}\right)=D(\mathrm{n}-S H G)$. It implies they aren't antipodal super-vertices.

Proposition 8.13. Let n-SuperHyperGraph $n$-SHG $=\left(G_{n} \subseteq P^{n}(V), E_{n} \subseteq P^{n}(V)\right)$ be an odd super-cycle. Then every couple of super-vertices are super-resolving set.

\section{9}


Proof. Let $X_{n}$ and $X_{n}^{\prime}$ be couple of super-vertices. Thus, by Proposition (8.12), $X_{n}$ and $X_{n}^{\prime}$ aren't antipodal super-vertices. It implies for every given couple of super-vertices $T_{n}$ and $T_{n}^{\prime}$, I get either $d\left(X_{n}, T_{n}\right) \neq d\left(X_{n}, T_{n}^{\prime}\right)$ or $d\left(X_{n}^{\prime}, T_{n}\right) \neq d\left(X_{n}^{\prime}, T_{n}^{\prime}\right)$. Therefore, $T_{n}$ and $T_{n}^{\prime}$ are super-resolved by either $X_{n}$ or $X_{n}^{\prime}$. It induces the set $\left\{X_{n}, X_{n}^{\prime}\right\}$ is super-resolving set.

Proposition 8.14. Let n-SuperHyperGraph $n$-SHG $=\left(G_{n} \subseteq P^{n}(V), E_{n} \subseteq P^{n}(V)\right)$ be an odd cycle. Then super-resolving number is two.

Proof. Let $X_{n}$ and $X_{n}^{\prime}$ be couple of super-vertices. Thus, by Proposition (8.12), $X_{n}$ and $X_{n}^{\prime}$ aren't antipodal super-vertices. It implies for every given couple of super-vertices $T_{n}$ and $T_{n}^{\prime}$, I get either $d\left(X_{n}, T_{n}\right) \neq d\left(X_{n}, T_{n}^{\prime}\right)$ or $d\left(X_{n}^{\prime}, T_{n}\right) \neq d\left(X_{n}^{\prime}, T_{n}^{\prime}\right)$. Therefore, $T_{n}$ and $T_{n}^{\prime}$ are super-resolved by either $X_{n}$ or $X_{n}^{\prime}$. It induces the set $\left\{X_{n}, X_{n}^{\prime}\right\}$ is super-resolving set.

Corollary 8.15. Let $n$-SuperHyperGraph $n$-SHG $=\left(G_{n} \subseteq P^{n}(V), E_{n} \subseteq P^{n}(V)\right)$ be an odd cycle. Then super-resolving set contains couple of super-vertices.

Proof. By Proposition (8.14), super-resolving number is two. By Proposition (8.13), every couple of super-vertices form super-resolving set. Therefore, super-resolving set contains couple of super-vertices.

Corollary 8.16. Let $\mathcal{G}$ be a family of $n$-SuperHyperGraphs $n-S H G=\left(G_{n} \subseteq P^{n}(V), E_{n} \subseteq P^{n}(V)\right)$ which are odd super-cycles with common super-vertex set $G_{n}$. Then simultaneously super-resolving set contains couple of super-vertices and super-resolving number is two.

\section{Extended Results For n-SuperHyperGraph}

\subsection{Smallest Super-resolving Number}

Proposition 9.1. Let $n$-SuperHyperGraph $n$-SHG $=\left(G_{n} \subseteq P^{n}(V), E_{n} \subseteq P^{n}(V)\right)$ be a super-path. Then every super-leaf forms super-resolving set.

Proof. Let $L_{n}$ be a super-leaf. For every given a couple of super-vertices $X_{n}$ and $X_{n}^{\prime}, \mathrm{I}$ get $d\left(L_{n}, X_{n}\right) \neq d\left(L_{n}, X_{n}^{\prime}\right)$. Since if I reassign indexes to super-vertices such that every super-vertex $X_{n}^{\prime}$ and $L_{n}$ have $i$ super-vertices amid themselves. Thus $j \leq i$ implies

$$
d\left(L_{n}, X_{n}\right)+c=d\left(L_{n}, X_{n}^{\prime}\right) \equiv d\left(L_{n}, X_{n}\right)<d\left(L_{n}, X_{n}^{\prime}\right) .
$$

Therefore, by $d\left(L_{n}, X_{n}\right)<d\left(L_{n}, X_{n}^{\prime}\right)$, I get $d\left(L_{n}, X_{n}\right) \neq d\left(L_{n}, X_{n}^{\prime}\right) . X_{n}$ and $X_{n}^{\prime}$ are arbitrary so $L_{n}$ super-resolves any given couple of super-vertices $X_{n}$ and $X_{n}^{\prime}$ which implies $\left\{L_{n}\right\}$ is a super-resolving set.

Proposition 9.2. Let n-SuperHyperGraph $n$-SHG $=\left(G_{n} \subseteq P^{n}(V), E_{n} \subseteq P^{n}(V)\right)$ be a super-path. Then a set including every couple of super-vertices is super-resolving set.

Proof. Let $X_{n}$ and $X_{n}^{\prime}$ be a couple of super-vertices. For every given a couple of super-vertices $Y_{n}$ and $Y_{n}^{\prime}$, I get either

$$
d\left(X_{n}, Y_{n}\right) \neq d\left(X_{n}, Y_{n}^{\prime}\right)
$$

or

$$
d\left(X_{n}^{\prime}, Y_{n}\right) \neq d\left(X_{n}^{\prime}, Y_{n}^{\prime}\right) .
$$


Proposition 9.3. Let $n$-SuperHyperGraph $n$-SHG $=\left(G_{n} \subseteq P^{n}(V), E_{n} \subseteq P^{n}(V)\right)$ be a 701 super-path. Then an 1-set contains leaf is super-resolving set and super-resolving number is one.

Proof. There are two super-leaves. Consider $L_{n}$ is a given super-leaf. By n-SuperHyperGraph n-SHG $=\left(G_{n} \subseteq P^{n}(V), E_{n} \subseteq P^{n}(V)\right)$ is a super-path, there's only one number to be seen. With rearranging the indexes of super-vertices, $d\left(L_{n}, V_{n}\right)=i$. Further more, $d\left(L_{n}, V_{n}\right)=i \neq j=d\left(L_{n}, V_{n}^{\prime}\right)$. Therefore, $L_{n}$ super-resolves every given couple of super-vertices $V_{n}$ and $V_{n}^{\prime}$. It induces 1-set containing leaf is super-resolving set. Also, super-resolving number is one.

Corollary 9.4. Let $\mathcal{G}$ be a family of $n$-SuperHyperGraphs $n-S H G=\left(G_{n} \subseteq P^{n}(V), E_{n} \subseteq P^{n}(V)\right)$ are super-paths with common super-vertex set $G_{n}$ such that they've a common super-leaf. Then simultaneously super-resolving number is 1, 1-set contains common leaf, is simultaneously super-resolving set for $\mathcal{G}$.

Proof. By Proposition (9.3), common super-leaf super-resolves every given couple of super-vertices $X_{n}$ and $X_{n}^{\prime}$, simultaneously. Thus 1-set containing common super-leaf, is simultaneously super-resolving set. Also, simultaneously super-resolving number is one.

Proposition 9.5. Let $\mathcal{G}$ be a family of $n$-SuperHyperGraphs $n-S H G=\left(G_{n} \subseteq P^{n}(V), E_{n} \subseteq P^{n}(V)\right)$ are super-paths with common super-vertex set $G_{n}$ such that for every super-leaf $L_{n}$ from $n$-SHG, there's another $n-S H G \in \mathcal{G}$ such that $L_{n}$ isn't super-leaf. Then an 2-set contains every couple of super-vertices, is super-resolving set. An 2-set contains every couple of super-vertices, is optimal-super-resolving set. Optimal-super-resolving number is two.

Proof. Suppose $V_{n}$ is a given super-vertex. If there are two super-vertices $X_{n}$ and $Y_{n}$ such that $d\left(X_{n}, V_{n}\right) \neq d\left(Y_{n}, V_{n}\right)$, then $X_{n}$ super-resolves $X_{n}$ and $Y_{n}$ and the proof is done. If not, $d\left(X_{n}, V_{n}\right)=d\left(Y_{n}, V_{n}\right)$, but for every given super-vertex $V_{n}^{\prime}$,

$$
d\left(X_{n}, V_{n}^{\prime}\right) \neq d\left(Y_{n}, V_{n}^{\prime}\right) .
$$

Corollary 9.6. Let $\mathcal{G}$ be a family of $n$-SuperHyperGraphs $n-S H G=\left(G_{n} \subseteq P^{n}(V), E_{n} \subseteq P^{n}(V)\right)$ are super-paths with common super-vertex set $G_{n}$ such that they've no common super-leaf. Then an 2-set is simultaneously optimal-super-resolving set and simultaneously optimal-super-resolving number is 2.

Proof. By Corollary (9.4), common super-leaf forms a simultaneously optimal-super-resolving set but in this case, there's no common super-leaf. Thus by Proposition (9.5), an 2-set is optimal-super-resolving set for any n-SHG $\mathcal{G}$. Then an 2 -set is simultaneously optimal-super-resolving set. It induces simultaneously optimal-super-resolving number is 2 . So every 2 -set is simultaneously optimal-super-resolving set for $\mathcal{G}$.

\subsection{Largest Optimal-super-resolving Number}

Super- $t$-partite, super-bipartite, super-star, super-wheel are also studied and they get us two type-results as individual and family.

Proposition 9.7. Let $n$-SuperHyperGraph $n$-SHG $=\left(G_{n} \subseteq P^{n}(V), E_{n} \subseteq P^{n}(V)\right)$ be a super-t-partite. Then every set excluding couple of super-vertices in different parts whose cardinalities of them are strictly greater than one, is optimal-super-resolving set. 
Proof. Consider two super-vertices $X_{n}$ and $Y_{n}$. Suppose super-vertex $M_{n}$ has same part with either $X_{n}$ or $Y_{n}$. Without loosing the generality, suppose $M_{n}$ has same part with $X_{n}$ thus it doesn't have common part with $Y_{n}$. Therefore,

$$
d\left(M_{n}, X_{n}\right)=2 \neq 1=d\left(M_{n}, Y_{n}\right)
$$

Corollary 9.8. Let $n$-SuperHyperGraph $n$-SHG $=\left(G_{n} \subseteq P^{n}(V), E_{n} \subseteq P^{n}(V)\right)$ be a super-t-partite. Let $|V| \geq 3$. Then every $(|V|-2)$-set excludes two super-vertices from different parts whose cardinalities of them are strictly greater than one, is optimal-super-resolving set and optimal-super-resolving number is $|V|-2$.

Proof. By Proposition (9.7), every $(|V|-2)$-set excludes two super-vertices from different parts whose cardinalities of them are strictly greater than one, is optimal-super-resolving set. Since if $X_{n}$ and $Y_{n}$ are either in same part or in different parts, then, by any given super-vertex $W_{n}, d\left(W_{n}, X_{n}\right)=d\left(W_{n}, Y_{n}\right)$. Thus 1-set isn't super-resolving set. There are same arguments for a set with cardinality $\leq|V|-3$ when pigeonhole principle implies at least two super-vertices have same conditions concerning either being in same part or in different parts.

Corollary 9.9. Let $n$-SuperHyperGraph $n$-SHG $=\left(G_{n} \subseteq P^{n}(V), E_{n} \subseteq P^{n}(V)\right)$ be a super-bipartite. Let $|V| \geq 3$. Then every $(|V|-2)$-set excludes two super-vertices from different parts, is optimal-super-resolving set and optimal-super-resolving number is $|V|-2$.

Proof. Consider $X_{n}$ and $Y_{n}$ are excluded by a $(|V|-2)$-set. Let $M_{n}$ be a given super-vertex which is distinct from them. By n-SuperHyperGraph n-SHG $=\left(G_{n} \subseteq P^{n}(V), E_{n} \subseteq P^{n}(V)\right)$ is a super-bipartite, $M_{n}$ has a common part with either $X_{n}$ or $Y_{n}$ and not with both of them. It implies $d\left(X_{n}, M_{n}\right) \neq d\left(Y_{n}, M_{n}\right)$. Since if $M_{n}$ has a common part with $X_{n}$, then $d\left(X_{n}, M_{n}\right)=2 \neq 1=d\left(Y_{n}, M_{n}\right)$. And if $M_{n}$ has a common part with $Y_{n}$, then $d\left(X_{n}, M_{n}\right)=1 \neq 2=d\left(Y_{n}, M_{n}\right)$. Thus $M_{n}$ super-resolves $X_{n}$ and $Y_{n}$. If $W_{n}$ is another super-vertex which is distinct from them, then pigeonhole principle induces at least two super-vertices have same conditions concerning either being in same part or in different parts. It implies $(|V|-3)$-set isn't super-resolving set. It implies $(|V|-2)$-set excludes two super-vertices from different parts, is optimal-super-resolving set and optimal-super-resolving number is $|V|-2$.

Corollary 9.10. Let $n$-SuperHyperGraph $n$-SHG $=\left(G_{n} \subseteq P^{n}(V), E_{n} \subseteq P^{n}(V)\right)$ be a super-star. Then every $(|V|-2)$-set excludes super-center and a given super-vertex, is optimal-super-resolving set and optimal-super-resolving number is $(|V|-2)$.

Proof. Consider $X_{n}$ and $Y_{n}$ are excluded by a $(|V|-2)$-set. Let $M_{n}$ be a given super-vertex which is distinct from them. By n-SuperHyperGraph n- $S H G=\left(G_{n} \subseteq P^{n}(V), E_{n} \subseteq P^{n}(V)\right)$ is super-star, $M_{n}$ has a common part with either $X_{n}$ or $Y_{n}$ and not with both of them. It implies $d\left(X_{n}, M_{n}\right) \neq d\left(Y_{n}, M_{n}\right)$. Since if $M_{n}$ has a common part with $X_{n}$, then $d\left(X_{n}, M_{n}\right)=2 \neq 1=d\left(Y_{n}, M_{n}\right)$. And if $M_{n}$ has a common part with $Y_{n}$, then $d\left(X_{n}, M_{n}\right)=1 \neq 2=d\left(Y_{n}, M_{n}\right)$. Thus $M_{n}$-resolves $X_{n}$ and $Y_{n}$. If $W_{n}$ is another super-vertex which is distinct from them, then pigeonhole principle induces at least two super-vertices have same conditions concerning either being in same part or in different parts. It implies $(|V|-3)$-set isn't super-resolving set. Therefore, every $(|V|-2)$-set excludes two super-vertices from different parts, is optimal-super-resolving set and optimal-super-resolving number is $|V|-2$. 
Corollary 9.11. Let $n$-SuperHyperGraph $n$-SHG $=\left(G_{n} \subseteq P^{n}(V), E_{n} \subseteq P^{n}(V)\right)$ be a ${ }_{782}$ super-wheel. Let $|V| \geq 3$. Then every $(|V|-2)$-set excludes super-center and a given super-vertex, is optimal-super-resolving set and optimal-super-resolving number is $|V|-2$.

Proof. Consider $X_{n}$ and $Y_{n}$ are excluded by a $(|V|-2)$-set. Let $M_{n}$ be a given super-vertex which is distinct from them. By n-SuperHyperGraph n- $S H G=\left(G_{n} \subseteq P^{n}(V), E_{n} \subseteq P^{n}(V)\right)$ is a super-wheel., $M_{n}$ has a common part with either $X_{n}$ or $Y_{n}$ and not with both of them. It implies $d\left(X_{n}, M_{n}\right) \neq d\left(Y_{n}, M_{n}\right)$. Since if $X_{n}$ is super-center, then $d\left(X_{n}, M_{n}\right)=1 \neq 2=d\left(Y_{n}, M_{n}\right)$. And if $Y_{n}$ is super-center, then $d\left(X_{n}, M_{n}\right)=2 \neq 1=d\left(Y_{n}, M_{n}\right)$. Thus $M_{n}$ super-resolves $X_{n}$ and $Y_{n}$. If $W_{n}$ is another super-vertex which is distinct from them, then pigeonhole principle induces at least two super-vertices have same conditions concerning either being in same part (non-center super-vertices) or in different parts. It implies $(|V|-3)$-set isn't super-resolving set. Therefore, every $(|V|-2)$-set super-center and a given super-vertex, is optimal-super-resolving set and optimal-super-resolving number is $|V|-2$.

Super-t-partite, super-bipartite, super-star, super-wheel are also studied but they get us one type-result involving family of them.

Corollary 9.12. Let $\mathcal{G}$ be a family of $n$-SuperHyperGraphs $n$-SHG $=\left(G_{n} \subseteq P^{n}(V), E_{n} \subseteq P^{n}(V)\right)$ which are super-t-partite with common super-vertex set $G_{n}$. Let $|V| \geq 3$. Then simultaneously optimal-super-resolving number is $|V|-2$ and every $(|V|-2)$-set excludes two super-vertices from different parts, is simultaneously optimal-super-resolving set for $\mathcal{G}$.

Proof. By Corollary (9.8), every result hold for any given n-SuperHyperGraph n- $S H G=\left(G_{n} \subseteq P^{n}(V), E_{n} \subseteq P^{n}(V)\right)$ which are super-t-partite. Thus every result hold for any given n-SuperHyperGraph n-SHG $=\left(G_{n} \subseteq P^{n}(V), E_{n} \subseteq P^{n}(V)\right)$ which are super-t-partite, simultaneously. Therefore, simultaneously super-resolving number is $|V|-2$ and every $(|V|-2)$-set excludes two super-vertices from different parts, is simultaneously optimal-super-resolving set for $\mathcal{G}$.

Corollary 9.13. Let $\mathcal{G}$ be a family of n-SuperHyperGraphs $n-S H G=\left(G_{n} \subseteq P^{n}(V), E_{n} \subseteq P^{n}(V)\right)$ which are super-bipartite with common super-vertex set $G_{n}$. Let $|V| \geq 3$. Then simultaneously optimal-super-resolving number is $|V|-2$ and every $(|V|-2)$-set excludes two super-vertices from different parts, is simultaneously optimal-super-resolving set for $\mathcal{G}$.

Proof. By Corollary (9.9), every result hold for any given n-SuperHyperGraph n-SHG $=\left(G_{n} \subseteq P^{n}(V), E_{n} \subseteq P^{n}(V)\right)$ which are super-bipartite. Thus every result hold for any given n-SuperHyperGraph n-SHG $=\left(G_{n} \subseteq P^{n}(V), E_{n} \subseteq P^{n}(V)\right)$ which are super-bipartite, simultaneously. Therefore, simultaneously super-resolving number is $|V|-2$ and every $(|V|-2)$-set excludes two super-vertices from different parts, is simultaneously optimal-super-resolving set for $\mathcal{G}$.

Corollary 9.14. Let $\mathcal{G}$ be a family of $n$-SuperHyperGraphs $n$-SHG $=\left(G_{n} \subseteq P^{n}(V), E_{n} \subseteq P^{n}(V)\right)$ which are super-star with common super-vertex set $G_{n}$. Let $|V| \geq 3$. Then simultaneously optimal-super-resolving number is $|V|-2$ and every $(|V|-2)$-set excludes super-center and a given super-vertex, is simultaneously optimal-super-resolving set for $\mathcal{G}$.

Proof. By Corollary (9.10), every result hold for any given n-SuperHyperGraph n-SHG $=\left(G_{n} \subseteq P^{n}(V), E_{n} \subseteq P^{n}(V)\right)$ which are super-star. Thus every result hold for any given n-SuperHyperGraph n-SHG $=\left(G_{n} \subseteq P^{n}(V), E_{n} \subseteq P^{n}(V)\right)$ which are 
super-star, simultaneously. Therefore, simultaneously super-resolving number is $|V|-2$ and every $(|V|-2)$-set excludes super-center and a given super-vertex, is simultaneously optimal-super-resolving set for $\mathcal{G}$.

Corollary 9.15. Let $\mathcal{G}$ be a family of $n$-SuperHyperGraphs $n$-SHG $=\left(G_{n} \subseteq P^{n}(V), E_{n} \subseteq P^{n}(V)\right)$ which are super-wheel with common super-vertex set $G_{n}$. Let $|V| \geq 3$. Then simultaneously optimal-super-resolving number is $|V|-2$ and every $(|V|-2)$-set excludes super-center and a given super-vertex, is simultaneously optimal-super-resolving set for $\mathcal{G}$.

Proof. By Corollary (9.11), every result hold for any given n-SuperHyperGraph n-SHG $=\left(G_{n} \subseteq P^{n}(V), E_{n} \subseteq P^{n}(V)\right)$ which are super-star. Thus every result hold for any given n-SuperHyperGraph n-SHG $=\left(G_{n} \subseteq P^{n}(V), E_{n} \subseteq P^{n}(V)\right)$ which are super-star, simultaneously. Therefore, simultaneously super-resolving number is $|V|-2$ and every $(|V|-2)$-set excludes super-center and a given super-vertex, is simultaneously optimal-super-resolving set for $\mathcal{G}$.

\section{Optimal-super-coloring Number in n-SuperHyperGraph}

Proposition 10.1. Let $n$-SuperHyperGraphs $n$-SHG $=\left(G_{n} \subseteq P^{n}(V), E_{n} \subseteq P^{n}(V)\right)$ be a super-complete. Then optimal-super-coloring number is $|V|$.

Proof. It's complete. It means for any two members of $V$, there's at least two distinct super-vertices contain them. Every super-vertex has edge with at least $|V|-1$ super-vertices. Thus $|V|$ is optimal-super-coloring number. Since any given member of $V$ has different color in comparison to another member of $V$. Then optimal-super-coloring number is $|V|$.

Proposition 10.2. Let $n$-SuperHyperGraphs $n$-SHG $=\left(G_{n} \subseteq P^{n}(V), E_{n} \subseteq P^{n}(V)\right)$ be a super-path. Then optimal-super-coloring number is two.

Proof. With alternative colors, super-path has distinct color for every super-vertices which have one super-edge in common. Thus if $X_{n}$ and $Y_{n}$ are two super-vertices which have one super-edge in common, then $X_{n}$ and $Y_{n}$ have different color. Therefore, optimal-super-coloring number is two. The representative of colors are two given super-vertices which have at least one super-edge in common.

Proposition 10.3. Let n-SuperHyperGraphs $n$-SHG $=\left(G_{n} \subseteq P^{n}(V), E_{n} \subseteq P^{n}(V)\right)$ be an even super-cycle. Then optimal-super-coloring number is two.

Proof. Since even super-cycle has even super-vertices, with alternative coloring of super-vertices, the super-vertices which have common super-edge, have different colors. So optimal-super-coloring number is two.

Proposition 10.4. Let n-SuperHyperGraphs $n$-SHG $=\left(G_{n} \subseteq P^{n}(V), E_{n} \subseteq P^{n}(V)\right)$ be an odd super-cycle. Then optimal-super-coloring number is three.

Proof. With alternative coloring on super-vertices, at end, two super-vertices have same color, and they've same super-edge. So, optimal-super-coloring number is three.

Proposition 10.5. Let n-SuperHyperGraphs $n$-SHG $=\left(G_{n} \subseteq P^{n}(V), E_{n} \subseteq P^{n}(V)\right)$ be a super-star. Then optimal-super-coloring number is two. 
Proof. Super-center has common super-edge with every other super-vertex. So it has different color in comparison to other super-vertices. So one color has only one super-vertex which has that color. All other super-vertices have no common super-edge amid each other. Then they've same color. The representative of this color is a super-vertex which is distinct from super-center. The set of representative of colors has two representatives which are super-center and a given super-vertex which isn't super-center. Optimal-super-coloring number is two.

Proposition 10.6. Let $n$-SuperHyperGraphs $n$-SHG $=\left(G_{n} \subseteq P^{n}(V), E_{n} \subseteq P^{n}(V)\right)$ be a super-wheel such that it has even super-cycle. Then optimal-super-coloring number is Three.

Proof. Super-center has unique color. So it's only representative of this color. Other super-vertices form a super-cycle which assigns distinct colors to the super-vertices which have common super-edge with each other when the number of colors is two. So a color for super-center and two colors for other super-vertices, make super-wheel has distinct colors for super-vertices which have common super-edge. Hence, optimal-super-coloring number is Three.

Proposition 10.7. Let n-SuperHyperGraph $n$-SHG $=\left(G_{n} \subseteq P^{n}(V), E_{n} \subseteq P^{n}(V)\right)$ be a super-wheel such that it has odd super-cycle. Then optimal-super-coloring number is four.

Proof. Without super-center, other super-vertices form odd super-cycle. Odd super-cycle has optimal-super-coloring number which is three. Super-center has common super-edges with all other super-vertices. Thus super-center has different colors with all other super-vertices. Therefore, optimal-super-coloring number is four. Four representatives of colors form optimal-super-coloring number where one representative is super-center and other three representatives are from all other super-vertices. So, optimal-super-coloring number is four.

Proposition 10.8. Let n-SuperHyperGraph $n$-SHG $=\left(G_{n} \subseteq P^{n}(V), E_{n} \subseteq P^{n}(V)\right)$ be a super-complete and super-bipartite. Then optimal-super-coloring number is two.

Proof. Every given super-vertex has super-edge with all super-vertices from another part. So the color of every super-vertex which is in a same part is same. Hence, two parts implies two different colors. It induces optimal-super-coloring number is two. The any of all super-vertices in every part, identify the representative of every color.

Proposition 10.9. Let n-SuperHyperGraph $n$-SHG $=\left(G_{n} \subseteq P^{n}(V), E_{n} \subseteq P^{n}(V)\right)$ be a super-complete and super-t-partite. Then optimal-super-coloring number is $t$.

Proof. Every part has same color for its super-vertices. Optimal-super-coloring number is $t$. Every part introduces one super-vertex as a representative of its color.

Proposition 10.10. Let $n-S H G=\left(G_{n} \subseteq P^{n}(V), E_{n} \subseteq P^{n}(V)\right)$ be $n$-SuperHyperGraph. Then optimal-super-coloring number is 1 if and only if $n-S H G=\left(G_{n} \subseteq P^{n}(V), E_{n} \subseteq P^{n}(V)\right)$ is super-empty.

Proof. $(\Rightarrow)$. Let optimal-super-coloring number be 1. It implies there's no super-vertex which has same edge with a vertex. So there's no super-edge. Since n- $S H G=\left(G_{n} \subseteq P^{n}(V), E_{n} \subseteq P^{n}(V)\right)$ is n-SuperHyperGraph and $\mathrm{n}-S H G=\left(G_{n} \subseteq P^{n}(V), E_{n} \subseteq P^{n}(V)\right)$ is super-empty.

$(\Leftarrow)$. Let $\mathrm{n}-S H G=\left(G_{n} \subseteq P^{n}(V), E_{n} \subseteq P^{n}(V)\right)$ be n-SuperHyperGraph and super-empty. Hence there's no super-edge. It implies for every given super-vertex, there's no common super-edge. It induces there's only one color for super-vertices. 
Hence the representative of this color is chosen from $\left|G_{n}\right|$ super-vertices. Thus optimal-super-coloring number is 1 .

Proposition 10.11. Let $n-S H G=\left(G_{n} \subseteq P^{n}(V), E_{n} \subseteq P^{n}(V)\right)$ be $n$-SuperHyperGraph. Then optimal-super-coloring number is 2 if and only if $n$-SHG $=\left(G_{n} \subseteq P^{n}(V), E_{n} \subseteq P^{n}(V)\right)$ is both super-complete and super-bipartite.

Proof. $(\Rightarrow)$. Let optimal-super-coloring number be two. So every super-vertex has either one super-vertex or two super-vertices with a common super-edge. The number of colors are two so there are two sets which each set has the super-vertices which have same color. If two super-vertices have same color, then they don't have a common edge. So every set is a part in that, no super-vertex has common super-edge. The number of these sets is two. Hence there are two parts in each of them, every super-vertex has no common super-edge with other super-vertices. Since n-SHG $=\left(G_{n} \subseteq P^{n}(V), E_{n} \subseteq P^{n}(V)\right)$ is n-SuperHyperGraph, n-SHG $=\left(G_{n} \subseteq P^{n}(V), E_{n} \subseteq P^{n}(V)\right)$ is both super-complete and super-bipartite.

$(\Leftarrow)$. Assume n- $S H G=\left(G_{n} \subseteq P^{n}(V), E_{n} \subseteq P^{n}(V)\right)$ is n-SuperHyperGraph, n-SHG $=\left(G_{n} \subseteq P^{n}(V), E_{n} \subseteq P^{n}(V)\right)$ is both super-complete and super-bipartite. Then all super edges are amid two parts. Every part has the super-vertices which have no super-edge in common. So they're assigned to have same color. There are two parts. Thus there are two colors to assign to the super-vertices in that, the super-vertices with common super-edge, have different colors. It induces optimal-super-coloring number is 2.

Proposition 10.12. Let $n-S H G=\left(G_{n} \subseteq P^{n}(V), E_{n} \subseteq P^{n}(V)\right)$ be $n$-SuperHyperGraph. Then optimal-super-coloring number is $|V|$ if and only if $n-S H G=\left(G_{n} \subseteq P^{n}(V), E_{n} \subseteq P^{n}(V)\right)$ is super-complete.

Proof. $(\Rightarrow)$. Let optimal-super-coloring number be $|V|$. Thus $\left|G_{n}\right|$ colors are available. So any given super-vertex has $\left|G_{n}\right|$ super-vertices which have common super-edge with them and every of them have common super-edge with each other. It implies every super-vertex has $\left|G_{n}\right|$ super-vertices which have common super-edge with them. Since n-SHG $=\left(G_{n} \subseteq P^{n}(V), E_{n} \subseteq P^{n}(V)\right)$ is n-SuperHyperGraph, $S H G=\left(G_{n} \subseteq P^{n}(V), E_{n} \subseteq P^{n}(V)\right)$ is super-complete.

$(\Leftarrow)$. Suppose n- $S H G=\left(G_{n} \subseteq P^{n}(V), E_{n} \subseteq P^{n}(V)\right)$ is super-complete. Every vertex has $\left|G_{n}\right|$ super-vertices which have common super-edge with them. Since all possible super-edges are available, the minimum number of colors are $\left|G_{n}\right|$. Thus optimal-super-coloring number is $|V|$.

General bounds for optimal-super-coloring number are computed.

Proposition 10.13. Let $n-S H G=\left(G_{n} \subseteq P^{n}(V), E_{n} \subseteq P^{n}(V)\right)$ be n-SuperHyperGraph. Then optimal-super-coloring number is obtained from the number of super-vertices which is $\left|G_{n}\right|$ and optimal-super-coloring number is at most $|V|$.

Proof. When every super-vertex is a representative of each color, optimal-super-coloring number is the union of number of members of all super-vertices and it happens in optimal-super-coloring number of super-complete which is $|V|$. When all super-vertices have distinct colors, optimal-super-coloring number is $|V|$ and it's sharp for super-complete.

The relation amid optimal-super-coloring number and main parameters of n-SuperHyperGraph is computed.

Proposition 10.14. Let $n-S H G=\left(G_{n} \subseteq P^{n}(V), E_{n} \subseteq P^{n}(V)\right)$ be $n$-SuperHyperGraph. Then optimal-super-coloring number is at most $\Delta+1$ and at least 2 . 
Proof. n-SuperHyperGraph is super-nontrivial. So it isn't super-empty which induces there's no super-edge. It implies optimal-super-coloring number is two. Since optimal-super-coloring number is one if and only if $\mathrm{n}-S H G=\left(G_{n} \subseteq P^{n}(V), E_{n} \subseteq P^{n}(V)\right)$ is super-empty if and only if n-SHG $=\left(G_{n} \subseteq P^{n}(V), E_{n} \subseteq P^{n}(V)\right)$ is super-trivial. A super-vertex with degree $\Delta$, has $\Delta$ super-vertices which have common super-edges with them. If these super-vertices have no super-edge amid each other, then optimal-super-coloring number is two especially, super-star. If not, then in the case, all super-vertices have super-edge amid each other, optimal-super-coloring number is $\Delta+1$, especially, super-complete.

Proposition 10.15. Let $n-S H G=\left(G_{n} \subseteq P^{n}(V), E_{n} \subseteq P^{n}(V)\right)$ be $n$-SuperHyperGraph and super-r-regular. Then optimal-super-coloring number is at most $r+1$.

Proof. n-SHG $=\left(G_{n} \subseteq P^{n}(V), E_{n} \subseteq P^{n}(V)\right)$ is super-r-regular. So any of super-vertex has $r$ super-vertices which have common super-edge with it. If these super-vertices have no common super-edge with each other, for instance super-star, optimal-super-coloring number is two. But since the super-vertices have common super-edge with each other, optimal-super-coloring number is $r+1$, for instance, super-complete.

\section{Applications in Time Table and Scheduling in Neutrosophic n-SuperHyperGraph}

Designing the programs to achieve some goals is general approach to apply on some issues to function properly. Separation has key role in the context of this style. Separating the duration of work which are consecutive, is the matter and it has important to avoid mixing up.

Step 1. (Definition) Time table is an approach to get some attributes to do the work fast and proper. The style of scheduling implies special attention to the tasks which are consecutive.

Step 2. (Issue) Scheduling of program has faced with difficulties to differ amid consecutive section. Beyond that, sometimes sections are not the same.

\subsection{First Case}

Step 3. (Model) As Figure (7), the situation is designed as a model. The model uses data to assign every section and to assign to relation amid section, three numbers belong unit interval to state indeterminacy, possibilities and determinacy. There's one restriction in that, the numbers amid two sections are at least the number of the relation amid them. Table (2), clarifies about the assigned numbers to these situation.

Table 1. Scheduling concerns its Subjects and its Connections as a n-SuperHyperGraph in a Model.

\begin{tabular}{c|lcc}
\hline Sections of $N H G$ & $n_{1}$ & $n_{2} \cdots$ & $n_{9}$ \\
\hline Values & $(0.99,0.98,0.55)$ & $(0.74,0.64,0.46) \cdots$ & $(0.99,0.98,0.55)$ \\
Connections of $N H G$ & $E_{1}$ & $E_{2}$ & $E_{3}$ \\
Values & $(0.01,0.01,0.01)$ & $(0.01,0.01,0.01)$ & $(0.01,0.01,0.01)$ \\
\hline
\end{tabular}




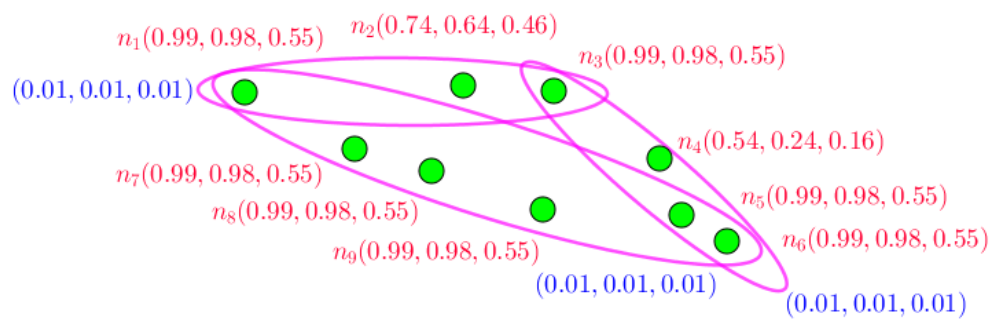

$N H G$

Figure 7. super-vertices are suspicions about choosing them.

\subsection{Second Case}

Step 4. (Solution) As Figure (7) shows, super hyper graph as model, proposes to use different types of coloring, resolving and dominating as numbers, sets, optimal numbers, optimal sets and et cetera.

(a) : The notions of dominating are applied.

$(i): n_{1}$ super-dominates every super-vertex from the set of super-vertices $\left\{n_{7}, n_{8}, n_{9}, n_{2}, n_{3}\right\} . n_{4}$ super-dominates every super-vertex from the set of super-vertices $\left\{n_{6}, n_{5}, n_{3}\right\}$. $n_{4}$ doesn't super-dominate every super-vertex from the set of super-vertices $\left\{n_{1}, n_{2}, n_{7}, n_{8}, n_{9}\right\}$.

(ii) : $\left\{n_{1}, n_{3}\right\}$ is super-coloring set but $\left\{n_{1}, n_{4}\right\}$ is optimal-super-dominating set.

(iii) : $(1.53,1.22,0.71)$ is optimal-super-dominating number.

(b) : The notions of resolving are applied.

$(i): n_{1}$ super-resolves two super-vertices $n_{4}$ and $n_{6}$.

(ii) : $V \backslash\left\{n_{1}, n_{4}\right\}$ is super-resolves set but $V \backslash\left\{n_{2}, n_{4}, n_{9}\right\}$ is optimal-super-resolving set.

(iii) : $(5,94,6.36,3.3)$ is optimal-super-resolving number.

(c) : The notions of coloring are applied.

$(i): n_{1}$ super-colors every super-vertex from the set of super-vertices $\left\{n_{7}, n_{8}, n_{9}, n_{2}, n_{3}\right\} . n_{4}$ super-colors every super-vertex from the set of super-vertices $\left\{n_{6}, n_{5}, n_{3}\right\}$. $n_{4}$ doesn't super-dominate every super-vertex from the set of super-vertices $\left\{n_{1}, n_{2}, n_{7}, n_{8}, n_{9}\right\}$.

(ii) : $\left\{n_{1}, n_{5}, n_{7}, n_{8}, n_{9}, n_{6}, n_{4}\right\}$ is super-coloring set but $\left\{n_{1}, n_{5}, n_{7}, n_{8}, n_{2}, n_{4}\right\}$ is optimal-super-coloring set.

(iii) : $(5.24,4.8,2.82)$ is optimal-super-coloring number.

Step 3. (Model) As Figure (8), the situation is designed as a model. The model uses data to assign every section and to assign to relation amid section, three numbers belong unit interval to state indeterminacy, possibilities and determinacy. There's one restriction in that, the numbers amid two sections are at least the number of the relation amid them. Table (2), clarifies about the assigned numbers to these situation.

Step 4. (Solution) As Figure (8) shows, $N H G_{3,3,3}^{3}=(V, E, \sigma, \mu)$ is neutrosophic complete 3 -partite hypergraph as model, proposes to use different types of degree of vertices, degree of hyperedges, co-degree of vertices, co-degree of hyperedges,
1024 1025 1026 1027 1028 


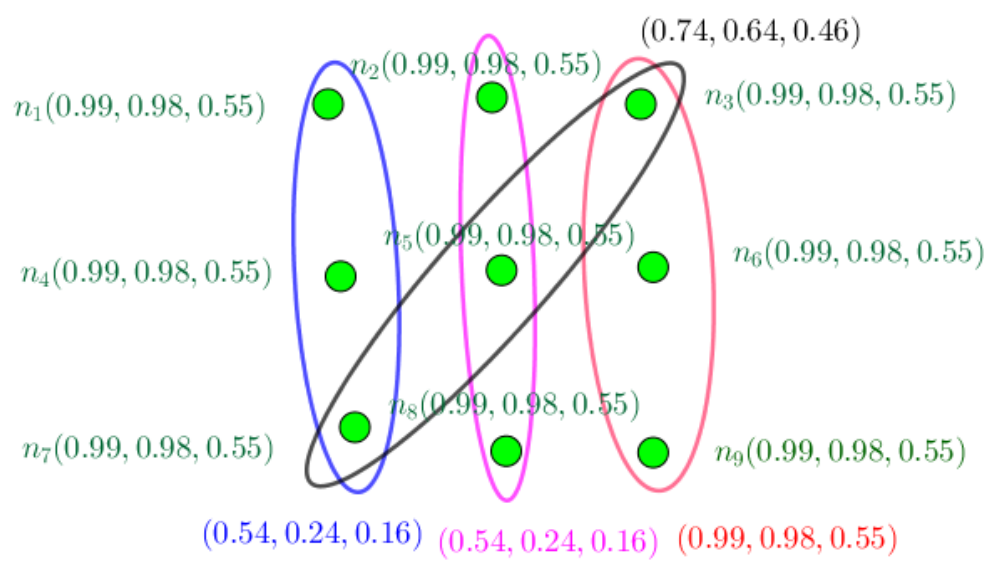

$N H G_{3,3,3}^{3}$

Figure 8. Vertices are suspicions about choosing them.

Table 2. Scheduling concerns its Subjects and its Connections as a Neutrosophic Hypergraph in a Model.

\begin{tabular}{c|lcc}
\hline Sections of $N H G$ & $n_{1}$ & $n_{2} \cdots$ & $n_{9}$ \\
\hline Values & $(0.99,0.98,0.55)$ & $(0.74,0.64,0.46) \cdots$ & $(0.99,0.98,0.55)$ \\
Connections of $N H G$ & $E_{1}, E_{2}$ & $E_{3}$ & $E_{4}$ \\
Values & $(0.54,0.24,0.16)$ & $(0.99,0.98,0.55)$ & $(0.74,0.64,0.46)$ \\
\hline
\end{tabular}

(i) : The notions of neutrosophic number are applied on vertices and hyperedges. ${ }^{1034}$ (a): A neutrosophic number of vertices $n_{1}, n_{2}, n_{3}$ is

$$
\Sigma_{i=1}^{3} \sigma\left(n_{i}\right)=(2.97,2.94,1.65) .
$$

(b) : A neutrosophic number of hyperedges $e_{1}, e_{2}, e_{3}$ is

$$
\Sigma_{i=1}^{3} \sigma\left(e_{i}\right)=(1.82,1.12,0.78) \text {. }
$$

where $e_{1}=(0.54,0.24,0.16), e_{2}=(0.74,0.64,0.46), e_{3}=(0.54,0.24,0.16) . \quad{ }_{1035}$

(ii) : The notions of degree, co-degree, neutrosophic degree and neutrosophic $\quad{ }_{1036}$ co-degree are applied on vertices and hyperedges.

(a) : A degree of any vertex $n_{1}, n_{2}, n_{4}, n_{6}, n_{8}, n_{9}$ is 1 and degree of any vertex $n_{3}, n_{5}, n_{7}$ is 2 .

(b) : A neutrosophic degree of vertex $n_{1}, n_{2}, n_{4}, n_{6}, n_{8}, n_{9}$ is $(0.99,0.98,0.55)$ and degree of any vertex $n_{3}, n_{5}, n_{7}$ is $(1.98,1.96,1.1)$.

(c) : A degree of any hyperedge is 3 .

$(d)$ : A neutrosophic degree of hyperedge is $(2.97,2.94,1.65)$.

(e) : A co-degree of vertices $n_{1}, n_{4}$ is 1 .

$(f)$ : A neutrosophic co-degree of vertices $n_{1}, n_{4}$ is $(0.54,0.24,0.16)$.

$(g)$ : A co-degree of hyperedges $e_{1}, e_{2}$ where $\mu\left(e_{1}\right)=(0.99,0.98,0.55)$ and $\mu\left(e_{2}\right)=(0.54,0.24,0.16)$ is 1 .

$(h)$ : A neutrosophic co-degree of hyperedges $e_{1}, e_{2}$ where $\mu\left(e_{1}\right)=(0.99,0.98,0.55)$ and $\mu\left(e_{2}\right)=(0.54,0.24,0.16)$ is $(0.99,0.98,0.55)$. 


\section{Open Problems}

The three notions of coloring, resolving and dominating are introduced on n-SuperHyperGraph. Thus,

Question 12.1. Is it possible to use other types super-edges to define different types of ${ }^{1054}$ coloring, resolving and dominating on n-SuperHyperGraph?

Question 12.2. Are existed some connections amid the coloring, resolving and dominating inside this concept and external connections with other types of coloring, resolving and dominating on n-SuperHyperGraph?

Question 12.3. Is it possible to construct some classes on n-SuperHyperGraph which have "nice" behavior?

Question 12.4. Which applications do make an independent study to apply these three types coloring, resolving and dominating on n-SuperHyperGraph?

Problem 12.5. Which parameters are related to this parameter?

Problem 12.6. Which approaches do work to construct applications to create independent study?

Problem 12.7. Which approaches do work to construct definitions which use all three definitions and the relations amid them instead of separate definitions to create independent study?

\section{Conclusion and Closing Remarks}

This study uses mixed combinations of different types of definitions, including coloring, resolving and dominating to study on n-SuperHyperGraph. The connections of super-vertices which are clarified by general super-edges differ them from each other and put them in different categories to represent one representative for each color, resolver and dominator. Further studies could be about changes in the settings to compare this notion amid different settings of n-SuperHyperGraph theory. One way is finding some relations amid three definitions of notions to make sensible definitions. In Table (3), some limitations and advantages of this study is pointed out.

Table 3. A Brief Overview about Advantages and Limitations of this study

\begin{tabular}{|c|c|}
\hline Advantages & Limitations \\
\hline 2. Defining (Dual) Dimension & 1. General Results \\
3. Defining (Dual) Coloring & 2. Connections Amid New Notions \\
4. Applying on Individuals & \\
5. Applying on Family & 3. Connections of Results \\
\hline
\end{tabular}

\section{References}

1. M. Akram, and G. Shahzadi, "Operations on Single-Valued Neutrosophic Graphs", Journal of uncertain systems 11 (1) (2017) 1-26. 
2. K. Atanassov, "Intuitionistic fuzzy sets", Fuzzy Sets Syst. 20 (1986) 87-96.

3. S. Broumi, M. Talea, A. Bakali and F. Smarandache, "Single-valued neutrosophic graphs", Journal of New Theory 10 (2016) 86-101.

4. N. Shah, and A. Hussain, "Neutrosophic soft graphs", Neutrosophic Set and Systems 11 (2016) 31-44.

5. Henry Garrett, "Chromatic Number and Neutrosophic Chromatic Number", Preprints 2021, 2021120177 (doi: 10.20944/preprints202112.0177.v1).

6. Henry Garrett, "Co-degree and Degree of classes of Neutrosophic Hypergraphs", Preprints 2022, 2022010027 (doi: 10.20944/preprints202201.0027.v1).

7. Henry Garrett, "Different Types of Neutrosophic Chromatic Number", Preprints 2021, 2021120335 (doi: 10.20944/preprints202112.0335.v1).

8. Henry Garrett, "Dimension and Coloring alongside Domination in Neutrosophic Hypergraphs", Preprints 2021, 2021120448 (doi:

10.20944/preprints202112.0448.v1).

9. Henry Garrett, "Metric Dimensions Of Graphs \#12", ResearchGate 2021 (doi: 10.13140/RG.2.2.20690.48322).

10. Henry Garrett, "Three Types of Neutrosophic Alliances based on Connectedness and (Strong) Edges", Preprints 2022, 2022010239 (doi:

10.20944/preprints202201.0239.v1).

11. Henry Garrett, "Neutrosophic Chromatic Number Based on Connectedness", Preprints 2021, 2021120226 (doi: 10.20944/preprints202112.0226.v1).

12. A. Shannon and K.T. Atanassov, "A first step to a theory of the intuitionistic fuzzy graphs", Proceeding of FUBEST (Lakov, D., Ed.) Sofia (1994) 59-61.

13. F. Smarandache, "A Unifying field in logics neutrosophy: Neutrosophic probability, set and logic, Rehoboth: "American Research Press (1998).

14. F. Smarandache, "Extension of HyperGraph to n-SuperHyperGraph and to Plithogenic n-SuperHyperGraph, and Extension of HyperAlgebra to n-ary (Classical-/Neutro-/Anti-) HyperAlgebra" Neutrosophic Sets and Systems 33 (2020) 290-296 (doi: 10.5281/zenodo.3783103).

15. H. Wang, F. Smarandache, Y. Zhang, and R. Sunderraman, "Single-valued neutrosophic sets", Multispace and Multistructure 4 (2010) 410-413.

16. L. A. Zadeh, "Fuzzy sets", Information and Control 8 (1965) 338-353. 